\title{
A Simplified IDA-PBC Design for Underactuated Mechanical Systems with Applications
}

\author{
Mutaz Ryalat ${ }^{a}$,Dina Shona Laila ${ }^{a}$ \\ ${ }^{a}$ School of Engineering Sciences, University of Southampton, Highfield, \\ Southampton SO17 1BJ, UK.
}

\begin{abstract}
We develop a method to simplify the partial differential equations (PDEs) associated to the potential energy for interconnection and damping assignment passivity based control (IDA-PBC) of a class of underactuated mechanical systems (UMSs). Solving the PDEs, also called the matching equations, is the main difficulty in the construction and application of the IDA-PBC. We propose a simplification to the potential energy PDEs through a particular parametrization of the closed-loop inertia matrix that appears as a coupling term with the inverse of the original inertia matrix. The parametrization accounts for kinetic energy shaping, which is then used to simplify the potential energy PDEs and their solution that is used for the potential energy shaping. This energy shaping procedure results in a closed-loop UMS with a modified energy function. This approach avoids the cancellation of nonlinearities, and extends the application of this method to a larger class of systems, including separable and non-separable port-controlled Hamiltonian (PCH) systems. Applications to the inertia wheel pendulum and the rotary inverted pendulum are presented, and some realistic simulations are presented which validate the proposed control design method and prove that global stabilization of these systems can be achieved. Experimental validation of the proposed method is demonstrated using a laboratory set-up of the rotary pendulum. The robustness of the closed-loop system with respect to external disturbances is also experimentally verified.
\end{abstract}

Key words: Hamiltonian systems, inertia wheel pendulum, nonlinear control, passivity-based control, rotary inverted pendulum, underactuated systems.

\footnotetext{
* A preliminary version of this paper has appeared in in the 2013 IEEE Conference on Decision and Control, Florence, Italy [28]. M. Ryalat acknowledges the support of The German-Jordanian University for his PhD scholarship to carry out this research.

Email addresses: mmr3g11@soton.ac.uk (Mutaz Ryalat), d.laila@soton.ac.uk (Dina Shona Laila).
}

Preprint submitted to European Journal of Control 3 September 2015 


\section{Introduction}

Control of underactuated mechanical systems (UMSs) has been a central and challenging topic that has attracted a lot of interest. UMSs, defined as systems that have fewer control inputs than the degrees of freedom to be controlled, can model many interesting applications including robotics, spacecraft, and satellites as well as a benchmark to study complex nonlinear control systems. While the absence of actuation in some degrees of freedom imposes a challenging task to achieve the desired control objectives with a lower number of actuators, underactuation control has the advantages of reducing the cost and complexity of the control system, and ensuring the functionality of a system in the case of actuation failure [22]. However, the fact that UMSs have complex internal dynamics and are not fully feedback linearizable complicates the control design, because the nonlinear control methods proposed for general mechanical systems can not be applied directly to this class of systems.

Various nonlinear control techniques have been developed for stabilization of UMSs (see [19] for a survey and [22] for a discussion). Among the most popular techniques, Passivity-based control methods such as controlled Lagrangian [9] and the interconnection and damping assignment passivity based control (IDA-PBC) [26] have proven to be powerful techniques due to their systematic and structure preserving approach, and the fact that they capture the essential physical property of energy conservation (passivity) $[7,26]$. A constructive stabilization method for a class of UMSs based on a newly developed immersion and invariance (I\&I) technique has been proposed in [30], and a comparison between the IDA-PBC and I\&I methods for UMSs has been presented in [16].

IDA-PBC is a control design method formulated for systems described by port-controlled Hamiltonian (PCH) models. The main idea of this method is to assign a new (desired) closed-loop PCH model that have certain features, utilizing the physically-inspired principles of energy shaping, interconnection structure and damping assignment (dissipation) [2]. The stabilization of an UMS via IDA-PBC is usually achieved by shaping the kinetic and potential energy functions and obtained through a state feedback law. The existence of such law constitutes the matching conditions of the IDA-PBC method which are a set of partial differential equations (PDEs) [25].

Solving these PDEs which identify the desired potential and kinetic energy functions is the main obstacle in the applicability of the IDA-PBC method. A number of constructive approaches to solve or simplify these PDEs for different classes of UMSs has been recently proposed in $[2,7,14,20,25,38]$ and references therein. Also, IDA-PBC has been applied to various underactuated systems, such as pendulum on a cart [35], inertia wheel and ball and beam systems [25], Pendubot [29] and Acrobot [20]. 
In this paper, we develop a constructive strategy to simplify and solve the PDEs of IDA-PBC method for a class of UMSs with underactuation degree one. The key idea is to parametrize the desired inertia matrix that shapes the kinetic energy, and used it to simplify the potential energy PDEs, and solve them to shape the potential energy function, thus achieving total energy shaping. That is, with suitable choice of the desired inertia matrix that must be positive definite and in spirit of some physical considerations, the potential energy PDE is simplified and its solution, the energy function must have a minimum at the desired equilibrium point. Furthermore, asymptotic stability is achieved by means of damping injection. This strategy expands the class of UMSs that can be dealt with compared to those in [2]. That is, we have proposed some design methods to deal with two groups of underactuated $\mathrm{PCH}$ systems, namely, the separable PCH systems (systems with constant inertia matrix) and non-separable PCH systems (systems with non-constant inertia matrix). We apply our result to a stabilization, as well as the swing up, of an inertia wheel pendulum and a rotary inverted pendulum (Furuta pendulum) systems. The later has relatively more complex dynamics than those of most other commonly studied benchmark systems [22].

The main contributions of the paper are:

- A constructive method to solve the potential energy PDEs for mechanical systems with underactuation degree one. The motivation of this is because most position stabilization problems can be solved by shaping the potential energy function [2]. For underactuated mechanical systems, kinetic energy function also needs to be shaped. We have assigned the inertia matrix that shapes the kinetic energy, and used it to simplify the potential energy PDE, and solve them to shape the potential energy function, thus reshaping the total energy function. While most works in literature use either normalized, linearized, or partial feedback linearized model of the UMS to simplify the problem, we have employed a full nonlinear model of the system.

- The proof that our proposed controller design method ensures an "almost" global asymptotic stabilization for the rotary inverted pendulum in its full nonlinear dynamics. To the best of our knowledge, this is the first work that achieves stabilization of this system over the entire domain of attraction using IDA-PBC. Also, it shows an "almost" global stabilization of the inertia wheel pendulum using realistic model parameters.

- A successful implementation of the controller to balance a laboratory rotary inverted pendulum hardware. The application is modified to include a friction compensator which is excluded throughout the $\mathrm{PCH}$ modelling.

- Robustness properties of our simplified IDA-PBC design with respect to external disturbances are experimentally verified. That is, we prove that the stability of the rotary inverted pendulum is preserved, for a certain margin, with respect to external disturbances. 
Definitions and notations. The set of real and natural numbers (including 0 ) are denoted respectively by $\mathbb{R}$ and $\mathbb{N}$. Given an arbitrary matrix $G$, we denote the transpose and the pseudo inverse of $G$ by $G^{\top}$ and $G^{+}$, respectively. $G^{\perp}$ denotes the full rank left annihilator of $G$, i.e. $G^{\perp} G=0$. We denote an $n \times n$ identity matrix with $I_{n}$. For any continuous function $H(i, j)$, we define $\nabla_{i} H(i, j):=\partial H(i, j) / \partial i . e_{i}, i \in n:=\{1, \cdots, n\}$ is the Euclidean basis vectors. We use a standard stability and passivity definitions for nonlinear systems [15]. The arguments of functions are often dropped whenever they are clear from the context.

\section{Problem Formulation}

We review the general procedure of the IDA-PBC design as has been proposed for instance in $[26,24]$. Some existing methods to solve the matching equations associated with the IDA-PBC are also reviewed, highlighting some limitations of those methods. Consider a PCH systems whose dynamics can be written as

$$
\begin{aligned}
{\left[\begin{array}{c}
\dot{q} \\
\dot{p}
\end{array}\right] } & =\left[\begin{array}{cc}
0 & I_{n} \\
-I_{n} & 0
\end{array}\right]\left[\begin{array}{c}
\nabla_{q} H \\
\nabla_{p} H
\end{array}\right]+\left[\begin{array}{c}
0 \\
G(q)
\end{array}\right] u, \\
y & =G^{\top}(q) \nabla_{p} H,
\end{aligned}
$$

where $q \in \mathbb{R}^{n}, p \in \mathbb{R}^{n}$ are the states and $u, y \in \mathbb{R}^{m}, m \leq n$, are the control input and the output, respectively. If $m=n$ the system is called fully-actuated, while if $m<n$ it is called underactuated. The Hamiltonian function, which is the total energy of the system, is defined as the sum of the kinetic energy and the potential energy

$$
H(q, p)=K(q, p)+V(q)=\frac{1}{2} p^{T} M^{-1}(q) p+V(q)
$$

where $M(q)>0$ is the symmetric inertia matrix and $V(q)$ is the potential energy function. IDA-PBC consists of two parts, which correspond to its design steps; the energy shaping and the damping injection, i.e.

$$
u=u_{e s}+u_{d i}
$$

\section{Energy shaping}

The main objective of IDA-PBC is to stabilize the PCH system by statefeedback controller. This is achieved by replacing the interconnection matrix and the energy function (Hamiltonian) of the system with a desired ones while preserving the $\mathrm{PCH}$ form of the total system in closed-loop. This can be mathematically expressed as

$$
\left[\begin{array}{cc}
0 & I_{n} \\
-I_{n} & 0
\end{array}\right]\left[\begin{array}{c}
\nabla_{q} H \\
\nabla_{p} H
\end{array}\right]+\left[\begin{array}{c}
0 \\
G(q)
\end{array}\right] u_{e s}=\left[\begin{array}{cc}
0 & M^{-1} M_{d} \\
-M_{d} M^{-1} & J_{2}(q, p)
\end{array}\right]\left[\begin{array}{l}
\nabla_{q} H_{d} \\
\nabla_{p} H_{d}
\end{array}\right] .
$$


The desired total energy in closed-loop is assigned to be

$$
H_{d}(q, p)=K_{d}(q, p)+V_{d}(q)=\frac{1}{2} p^{T} M_{d}^{-1}(q) p+V_{d}(q)
$$

with $M_{d}=M_{d}^{T}>0$ the desired inertia matrix and $V_{d}(q)$ the desired potential energy, such that $H_{d}$ has an isolated minimum at the desired equilibrium point $q_{e}$, i.e.

$$
q_{e}=\arg \min H_{d}(q)=\arg \min V_{d}(q)
$$

The following conditions are required so that (6) holds:

Condition 2.1 Necessary extremum assignment: $\nabla_{q} V_{d}\left(q_{e}\right)=0$.

Condition 2.2 Sufficient minimum assignment: $\nabla_{q}^{2} V_{d}\left(q_{e}\right)>0$, i.e. the Hessian of the function at the equilibrium point is positive.

Equation (4) constitutes the matching conditions of the IDA-PBC method [26], which is a set of PDEs in the form of

$$
G^{\perp}\left\{\nabla_{q} H-M_{d} M^{-1} \nabla_{q} H_{d}+J_{2} M_{d}^{-1} p\right\}=0,
$$

with $J_{2}=-J_{2}^{T}$ a free parameter. PDEs (7) can be separated into two elements; kinetic energy PDEs (dependent on $p$ ):

$$
G^{\perp}\left\{\nabla_{q}\left(p^{T} M^{-1} p\right)-M_{d} M^{-1} \nabla_{q}\left(p^{T} M_{d}^{-1} p\right)+2 J_{2} M_{d}^{-1} p\right\}=0
$$

and potential energy PDEs (independent of $p$ ):

$$
G^{\perp}\left\{\nabla_{q} V-M_{d} M^{-1} \nabla_{q} V_{d}\right\}=0 .
$$

If these sets of PDEs (8) and (9) are solved, or in other words $M_{d}, V_{d}$ and $J_{2}$ are obtained, then $u_{e s}$ is given by

$$
\begin{aligned}
u_{e s} & =\left(G^{\top} G\right)^{-1} G^{\top}\left(\nabla_{q} H-M_{d} M^{-1} \nabla_{q} H_{d}+J_{2} M_{d}^{-1} p\right) \\
& =G^{+}\left(\nabla_{q} H-M_{d} M^{-1} \nabla_{q} H_{d}+J_{2} M_{d}^{-1} p\right) .
\end{aligned}
$$

\section{Damping injection}

The next task after finding $u_{e s}$ is to find the damping injection (dissipation) controller, which is

$$
u_{d i}=-K_{v} G^{T} \nabla_{p} H_{d}, \quad K_{v}>0
$$

to add the damping to the closed-loop system that ensures asymptotic stabilization to the desired equilibrium. $u_{d i}$ is applied via a negative feedback of the passive output to achieve asymptotic stability, provided that the system is zero-state detectable. The system (1) is called zero-state observable if 
$u(t)=y(t)=0, \forall t \geq 0 \Longrightarrow(q(t), p(t))=\left(q_{e}, 0\right)$. It is zero-state detectable if $u(t)=y(t)=0, \quad \forall t \geq 0 \Longrightarrow \lim _{t \rightarrow \infty}(q(t), p(t))=\left(q_{e}, 0\right)$.

Given a PCH system (1), by applying the IDA-PBC design we obtain the following preserved $\mathrm{PCH}$ dynamics

$$
\begin{aligned}
{\left[\begin{array}{c}
\dot{q} \\
\dot{p}
\end{array}\right] } & =\left[\begin{array}{cc}
0 & M^{-1} M_{d} \\
-M_{d} M^{-1} & J_{2}-R_{d}
\end{array}\right]\left[\begin{array}{c}
\nabla_{q} H_{d} \\
\nabla_{p} H_{d}
\end{array}\right] \\
y_{d} & =G^{\top}(q) \nabla_{p} H_{d},
\end{aligned}
$$

where $R_{d}=G K_{v} G^{\top}>0$ is the dissipation matrix.

\subsection{Total energy shaping}

From the passivity-based control perspective, the natural way to stabilize a mechanical system is by modifying/shaping its energy function consisting of the kinetic and the potential functions. The most important step in the design procedure is to shape its potential energy function. This is due to several reasons; first, the stability of the system is achieved through the potential energy shaping $[18,36]$. That is, the energy of the mechanical system in its balancing position is represented by the potential energy at the coordinates $q_{n}$ [32]. Second, the qualitative behavior of the system can be concluded from the features of the potential energy function. Third, most approaches that relies on kinetic energy shaping only resulted in unsatisfactory stability and closed-loop performance of the system. That is, stability is limited to a reduced Domain of Attraction (DoA) or phase space. In $[8,9]$ where the method of controlled Lagrangian restricted to kinetic energy shaping has been used, only local asymptotic stability has been achieved (for instance in the inverted pendulum applications, only the upper hemisphere of the DoA).

As for the Hamiltonian framework, where the IDA-PBC is the most popular methodology used, the focus in most approaches has been on solving the kinetic energy PDEs, by modifying the interconnection matrix $M_{d}$ and used it to produce the closed-loop potential energy function $V_{d}$. As discussed in [2] (see Remark 7), the restriction on $M_{d}$ in solving the kinetic energy PDEs limits achieving global stability. This is evident by the reduced DoA obtained in the cart pendulum system and Furuta pendulum system applications in [2] and [38]. While the potential energy shaping is sufficient in most regulation problems for mechanical systems, for underactuated systems it is necessary also to shape the kinetic energy function, thus achieving total energy shaping that enlarges the class of systems that can be stabilized [25]. 


\subsection{The matching equations}

Some constructive techniques have been proposed in literature, for instance in $[2,7,14,38]$, to solve the matching equations for various subclasses of $\mathrm{PCH}$ systems, imposing particular conditions to satisfy. In [14], a method to reduce the kinetic energy PDEs (8) to a simpler nonlinear ODEs has been proposed. This method has been developed for a class of UMSs whose open-loop inertia matrix $M$ depends only on the non-actuated coordinate. The idea is then to parametrize the closed-loop inertia matrix $M_{d}$ to follow the structure of $M$ and to use the free parameter matrix $J_{2}$ to force these equations to satisfy certain mathematical properties and hence reducing these PDEs to several sets of nonlinear ODEs. This procedure contributes to kinetic energy shaping. The assigned $M_{d}$ which must be symmetric and positive definite is then substituted into (9) to solve for $V_{d}$, i.e. potential energy shaping.

Another technique to simplify the kinetic energy PDEs has been proposed in [7]. A notation of $\lambda-$ Equations originally proposed in [6] to triangulate a highly nonlinear system of PDEs into a set of linear PDEs has been adopted to generate one quadratic PDE in $\lambda$ and subsequently a linear PDE in $M_{d}$. This PDE has been solved and the resulting $M_{d}$ is used in (9) to solve for $V_{d}$.

In [2], a constructive technique to solve the PDEs has been suggested. In this method several conditions have been imposed on $M, G$ and $V$ to simplify these PDEs. First, the system has underactuation degree one and $M$ does not depend on the unactuated coordinate. This condition eliminates the first term (also called the forcing term) in (8) which transforms this equation from inhomogeneous to homogeneous PDEs. If the first condition is not satisfied, a partial feedback linearization can be used. Then, parametrizing $M_{d}$ and partially parametrizing $J_{2}$, in such a way so that the PDEs (8) are transformed into a set of algebraic equation in the (partially) unknown $J_{2}$ for a fixed $M_{d}$, will solve the kinetic energy shaping problem (see Proposition 3 of [2]). The potential energy shaping is then achieved by using the fixed $M_{d}$ and by imposing that $M_{d}$ and $V_{d}$ are both functions of one and the same actuated coordinate. Inspired by the work of [2], several IDA-PBC controllers have been proposed in [35], [29], [20] and [38], for various UMSs.

\subsection{Simplifying the PDEs via change of coordinates}

A common practice to simplify the PDEs is to employ a change of coordinates (see for instance [2], [25], [38] and references therein). In [25], a change of coordinates is used to simplify the description of the dynamics of the inertia wheel pendulum, which is a separable PCH system. In [38], the kinetic energy PDEs, which are nonlinear and inhomogeneous, is simplified via a certain change of coordinates that eliminates the forcing term in this set of PDEs, making them homogeneous. This change of coordinates involves replacing the momentum 
vector $p$ by its corresponding quasi-velocities. The method is known as quasilinearization as it involves elimination of the quadratic terms of the velocity (resembles the linearization). As a result, the inertia matrix becomes constant in the energy function i.e. the system is only affected by the potential field [38]. In [11] and [37], some necessary and sufficient conditions (such as, Riemannian curvature, constant inertia matrix, skew-symmetry, and zero Christoffel symbols) on the inertia matrix $M$ have been given, which need to be verified for the existence of such transformation. Although these methods simplify the control problem, the application is limited to the class of systems that admits quasi-linearization. For example, the method in [38] can not apply directly to the rotary inverted pendulum.

Inspired by the discussion above, we propose a novel approach to this control problem, concentrating our attention on simplifying and solving the potential energy PDEs which implies modifying the inertia matrix (notice that in (9), $M_{d}$ is the only term that can be modified). By parametrizing $M_{d}$ that shapes the kinetic energy function, one can use it to solve the potential energy PDEs rather than focusing on finding the solution for the kinetic energy PDEs itself.

\section{Main Results: Simplifying the Potential Energy PDEs}

In this section, we propose an alternative approach, focusing on solving (9) the set of PDEs associated with the potential energy. In [2], it was shown that the potential energy PDEs can be explicitly solved, provided that the inertia matrix $M$ and the potential energy $V$ depend only on the actuated coordinates. This method is applicable only to a subclass of underactuated mechanical systems that satisfy the following conditions:

Condition 3.1 The inertia matrix $M$ and the potential energy function $V$ do not depend on the unactuated coordinates.

Condition 3.2 The system has underactuation degree one, i.e. $m=n-1$.

Violating Condition 3.1, the forcing term $G^{\perp} \nabla_{q}\left(p^{T} M^{-1} p\right)$ in (8) will not be eliminated and hence does not simplify the process of solving the PDEs. In this work, we propose a new procedure to relax Condition 3.1 (while Condition 3.2 is kept), with the implication to also extend the subset of underactuated systems that can be treated using this method.

In the sequel, for the sake of the clarity of the constructive presentation, we will focus our attention to systems with two degrees of freedom (and underactuation degree one), i.e $n=2, m=1$. This is motivated by the fact that the majority of classical underactuated control problems, such as those examples mentioned in Section 1 (see also the survey paper [19] for most common examples of UMSs) share this property. However, extending our results to more 
general class of systems with underactuation degree 1 is possible, although the formulation will be more complicated. The following condition identifies the class of PCH systems that we consider in this paper:

Condition 3.3 The inertia matrix $M$ and the potential energy function $V$ depends only on one coordinate, not necessarily the actuated coordinate.

Condition 3.3 is a relaxation of Condition 3.1, in the sense that this method can be applied to all cases; either 1) $M$ is constant, 2) $M$ depends only on one, either actuated or unactuated, coordinate. Without loss of generality, we assume that the unactuated coordinate is $q_{1}$ and hence $G=e_{2}\left(G^{\perp}=e_{1}^{\top}\right)$, otherwise we may reorder the coordinates to come up with this structure.

Clearly, one source of difficulty in solving (9) arises from the complex structure and, for many systems, the dependencies on $q$ of the inertia matrix, and hence its inverse. Recognizing that (9) contains a coupling term $\left(M_{d} M^{-1}\right)$, we can simplify this PDE by choosing $M_{d}$ with certain structure to allow the elimination of some terms as follows.

Let the inertia matrix

$$
M(q)=\left[\begin{array}{ll}
k_{1}\left(q_{1}\right) & k_{2}\left(q_{1}\right) \\
k_{2}\left(q_{1}\right) & k_{3}\left(q_{1}\right)
\end{array}\right]
$$

Denote its determinant as $\Delta:=\operatorname{det}(M)=k_{1}\left(q_{1}\right) k_{3}\left(q_{1}\right)-k_{2}^{2}\left(q_{1}\right)$. By the inclusion of $\Delta$ in the desired inertia matrix $M_{d}$, or in other words by choosing

$$
M_{d}(q)=\left[\begin{array}{ll}
m_{1}(q) & m_{2}(q) \\
m_{2}(q) & m_{3}(q)
\end{array}\right]=\Delta\left[\begin{array}{ll}
\bar{m}_{1}(q) & \bar{m}_{2}(q) \\
\bar{m}_{2}(q) & \bar{m}_{3}(q)
\end{array}\right]
$$

and suppose that Conditions 3.2 and 3.3 hold, (9) can then be written as

$$
\left[\begin{array}{ll}
1 & 0
\end{array}\right]\left\{\left[\begin{array}{c}
\nabla_{q_{1}} V\left(q_{1}\right) \\
0
\end{array}\right]-\left[\begin{array}{c}
\bar{m}_{1} \Delta \bar{m}_{2} \Delta \\
\bar{m}_{2} \Delta \bar{m}_{3} \Delta
\end{array}\right]\left[\begin{array}{cc}
\frac{k_{3}}{\Delta} & \frac{-k_{2}}{\Delta} \\
\frac{-k_{2}}{\Delta} & \frac{k_{1}}{\Delta}
\end{array}\right]\left[\begin{array}{c}
\nabla_{q_{1}} V_{d}(q) \\
\nabla_{q_{2}} V_{d}(q)
\end{array}\right]\right\}=0
$$

which further gives

$$
\left(\bar{m}_{1} k_{3}-\bar{m}_{2} k_{2}\right) \nabla_{q_{1}} V_{d}(q)+\left(-\bar{m}_{1} k_{2}+\bar{m}_{2} k_{1}\right) \nabla_{q_{2}} V_{d}(q)=\nabla_{q_{1}} V\left(q_{1}\right) .
$$

Notice that with this parametrization of $M_{d}, \Delta$ is eliminated from the potential energy PDE, which gives the first step of the simplification.

In general, based on the forms of the inertia matrix, PCH systems, thus in our case the UMSs, can be classified into two groups [17]: I) separable UMSs, if the inertia matrix is constant i.e. $M$ is independent of the states $(q, p), \mathbf{I I})$ nonseparable UMSs, if otherwise. Now, we will deal with each group separately. 


\subsection{Separable UMSs}

An example of separable UMSs is the inertia wheel pendulum (IWP) [34]. In some cases, a non-separable UMS model can be transformed into a separable one via partial feedback linearization [31] or a change of coordinates [22,37].

Because $M$ is constant, we can choose $M_{d}$ to be also constant. Hence, we can write (13) and (14) as

$$
\begin{gathered}
M=\left[\begin{array}{ll}
k_{1} & k_{2} \\
k_{2} & k_{3}
\end{array}\right], \\
M_{d}=\left[\begin{array}{ll}
m_{1} & m_{2} \\
m_{2} & m_{3}
\end{array}\right]=\Delta\left[\begin{array}{ll}
\bar{m}_{1} & \bar{m}_{2} \\
\bar{m}_{2} & \bar{m}_{3}
\end{array}\right] .
\end{gathered}
$$

Further simplification to (16) is achieved by choosing

$$
\bar{m}_{2}=\frac{k_{2}}{k_{1}} \bar{m}_{1}+\varepsilon, \quad \varepsilon>0
$$

yielding

$$
\begin{aligned}
\left(\bar{m}_{1} k_{3}-\bar{m}_{2} k_{2}\right) \nabla_{q_{1}} V_{d}(q)+\varepsilon k_{1} \nabla_{q_{2}} V_{d}(q) & =\nabla_{q_{1}} V\left(q_{1}\right), \\
\left(\bar{m}_{1}\left(k_{3}-\frac{k_{2}^{2}}{k_{1}}\right)-\varepsilon k_{2}\right) \nabla_{q_{1}} V_{d}(q)+\varepsilon k_{1} \nabla_{q_{2}} V_{d}(q) & =\nabla_{q_{1}} V\left(q_{1}\right) .
\end{aligned}
$$

The general solution of this PDE is of the form

$$
V_{d}(q)=V_{d}\left(q_{1}\right)+\Psi\left(q_{2}+\pi_{1} q_{1}\right)
$$

where $\pi_{1}$ is constant. The procedure can now be summarized in the following proposition.

Proposition 3.1 Consider the separable underactuated PCH system (1) satisfying Conditions 3.2 and 3.3. Let the inertia matrix $M>0$ and the parametrized desired inertia matrix $M_{d}>0$ take the form (17) and (18), respectively. Then the potential energy PDE (9) can be written in its simplified form (20) by choosing $\bar{m}_{2}=\frac{k_{2}}{k_{1}} \bar{m}_{1}+\varepsilon$, with a constant $\varepsilon>0$. Furthermore, the solution of the potential energy PDE is given by (21).

Remark 3.1 The choice of $\bar{m}_{2}=\frac{k_{2}}{k_{1}} \bar{m}_{1}+\varepsilon$ in the separable case is critical to make the potential energy PDE as simple as possible. The fact that $M$ is constant gives more freedom in parametrizing the matrix $M_{d}$ to assign $\bar{m}_{i}$, $i=1,2,3$, where again the parametrization is such that $M_{d}>0$ is symmetric and $V_{d}$ admits a minimum at the desired equilibrium point $q_{e}$. Furthermore, since $M$ and $M_{d}$ are constant, we can choose $J_{2}=0$. 


\subsection{Non-separable UMSs}

Non-separable UMSs are more complex. This class of systems contains the majority of UMSs that frequently appear in applications. We proceed with our simplification by choosing

$$
\bar{m}_{2}(q)=\frac{k_{2}\left(q_{1}\right)}{k_{1}\left(q_{1}\right)} \bar{m}_{1}(q)
$$

which simplifies (16) into

$$
\begin{aligned}
\left(\bar{m}_{1}(q) k_{3}\left(q_{1}\right)-\bar{m}_{2}(q) k_{2}\left(q_{1}\right)\right) \nabla_{q_{1}} V_{d}(q) & =\nabla_{q_{1}} V\left(q_{1}\right) \\
\bar{m}_{1}(q)\left(k_{3}\left(q_{1}\right)-\frac{k_{2}^{2}\left(q_{1}\right)}{k_{1}\left(q_{1}\right)}\right) \nabla_{q_{1}} V_{d}(q) & =\nabla_{q_{1}} V\left(q_{1}\right)
\end{aligned}
$$

which is then rewritten as

$$
\nabla_{q_{1}} V_{d}(q)=\frac{\nabla_{q_{1}} V\left(q_{1}\right)}{\bar{m}_{1}(q) \pi\left(q_{1}\right)}, \quad \pi\left(q_{1}\right)=\left(k_{3}-\frac{k_{2}^{2}}{k_{1}}\right) .
$$

The obvious next step is to find $\bar{m}_{1}(q)$ (notice that $\nabla_{q_{1}} V\left(q_{1}\right)$ and $\pi\left(q_{1}\right)$ are known) in (24) such that the solution of this potential energy PDE guarantees that $V_{d}$ has an isolated minimum. As $M$ is a function of $q_{1}$ only, we can simply take $M_{d}$ as also a function of $q_{1}$ only. Then, (24) can be solved as either ODE or PDE. However, we have solved it as PDE for two reasons; first, to satisfy Conditions 2.1 and 2.2, and second, to keep track on the coordinate $q_{2}$. The solution of (24) is given by

$$
V_{d}(q)=\int_{0}^{q_{1}} \frac{\nabla_{x} V(x)}{\bar{m}_{1}(x) \pi(x)} d x+\Psi\left(q_{2}\right)
$$

with $\Psi(\cdot)$ an arbitrary differentiable function that must be chosen to satisfy (6). This whole procedure can now be summarized in the following proposition.

Proposition 3.2 Consider the non-separable underactuated PCH system (1) satisfying Conditions 3.2 and 3.3. Let the inertia matrix $M(q)>0$ and the parametrized desired inertia matrix $M_{d}(q)>0$ take the form (13) and (14), respectively. The potential energy PDE (9) can then be written in its simplified form (24) by choosing $\bar{m}_{2}=\frac{k_{2}}{k_{1}} \bar{m}_{1}$. Furthermore, the solution of the potential energy PDE is given by (25).

Remark 3.2 Using Propositions 3.1 and 3.2, PDEs (8) and (9) are simplified and their general solutions depend on the dynamics of the underactuated mechanical system. Clearly, the inclusion of the determinant $\Delta$ is essential in the parametrization of $M_{d}$. It simplifies the PDEs by canceling out the term $\Delta$ from the denominator of each element of $M^{-1}$. 
Remark 3.3 The elimination of the second term on the left hand side of (16) by using (22) is critical to make the potential energy PDE as simple as possible. The parametrization of $M_{d}$ to assign $\bar{m}_{i}, i=1,2,3$ depends mainly on the dynamics of the system. However, the choice of $\bar{m}_{1}$ and subsequently $\bar{m}_{2}$ and $\bar{m}_{3}$ is not free; first, it should guarantee that $M_{d}$ is symmetric and positive definite (i.e. $\bar{m}_{1}>0$, and $\bar{m}_{3}>\frac{\bar{m}_{2}^{2}}{\bar{m}_{1}}$ ). Then among the set of possible $m_{i}$, they should be chosen such that $V_{d}$ satisfies Conditions 2.1 and 2.2. Once all are satisfied, $J_{2}(q, p)$ is brought into play.

Now, by Proposition 3.2, we have established the existence of a solution for the potential energy PDE. It remains to verify the existence of solution(s) to the kinetic energy PDEs (8), which is essential to complete the kinetic energy shaping, and to find $J_{2}$ that contributes to the last term on the right hand side of (10). As $M_{d}$ has now been fixed, the kinetic energy PDEs (8) is no longer a nonlinear and inhomogeneous PDE, but is an algebraic equation

$$
G^{\perp}\left\{2 J_{2} M_{d}^{-1} p\right\}=G^{\perp}\left\{M_{d} M^{-1} \nabla_{q} H_{d}-\nabla_{q} H\right\},
$$

that can be solved to obtain $J_{2}$.

Remark 3.4 The application of the result in [2] to a pendulum on a cart, which is a non-separable system, requires partial feedback linearization to satisfy Condition 3.1. Using Proposition 3.2, we can provide a solution directly without linearization. Furthermore, our proposed method is the first that guarantees an "almost" global asymptotic stability of the cart pendulum system using IDA-PBC. In Section 5 we will show as an application example a rotary inverted pendulum that has a similar but more complex dynamics than a pendulum on a cart. Note that for these type of systems, a global stabilization cannot be achieved as the systems evolve on manifolds which are not diffeomorphic to the euclidean space [3]

Equations (20) and (24) represent a simplified PDEs which are applicable to a wide range of UMSs such as an inertia wheel pendulum, a pendulum on a cart, and a rotary pendulum. Case studies are presented in Sections 4 and 5.

\section{Separable Hamiltonian Systems: the Inertia Wheel Pendulum Example}

In this section we apply the proposed design method to stabilize at the upright position, an inertia wheel pendulum (IWP), also known as a reaction wheel pendulum. IWP was first introduced in [34], where a control design based on a partial feedback linearization was proposed. Another approach based on global change of coordinates to transform the dynamics of the system into strict feedback form and then applying backstepping procedure was presented 
in [22]. Energy-based approach was used in [12]. IDA-PBC of IWP has been recently reported in [25], where a change of coordinates and scaling have been used. Here, our design method is applied without any change of coordinates or scaling, simulating a practical set-up using parameters from a real system.

\subsection{IWP model}

We use the model of the Quanser IWP module [10], whose simplified free body diagram of the mechanical part is shown in Figure 1. The system comprises an unactuated planar inverted pendulum with actuated symmetric wheel attached to the end of the pendulum and is free to rotate about an axis parallel to the axis of rotation of the pendulum. The system has two degrees-of-freedom; the angular position of the pendulum $q_{1}$ and the angular position of the wheel $q_{2}$. As only the wheel is actuated, the system is underactuated.

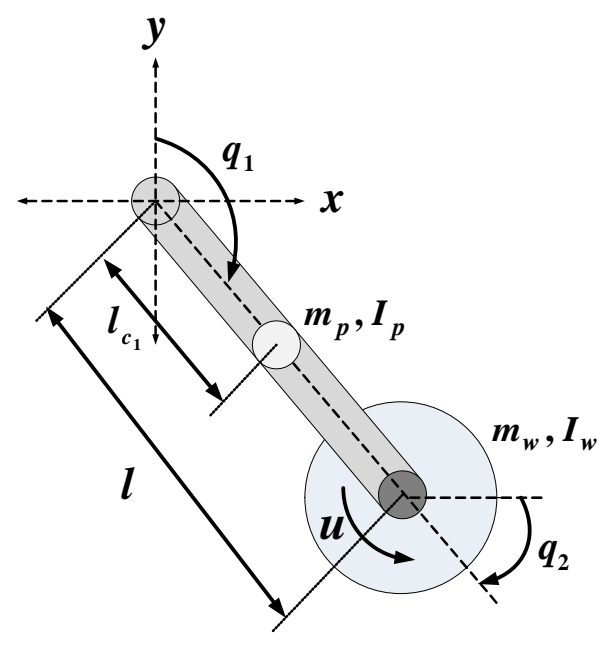

Fig. 1. The inertia wheel pendulum.

The Euler-Lagrange's equations of motion for the IWP are [25]:

$$
\left[\begin{array}{cc}
a_{1}+a_{2} & a_{2} \\
a_{2} & a_{2}
\end{array}\right]\left[\begin{array}{l}
\ddot{q}_{1} \\
\ddot{q}_{2}
\end{array}\right]+\left[\begin{array}{c}
-a_{3} \sin \left(q_{1}\right) \\
0
\end{array}\right]=\left[\begin{array}{l}
0 \\
1
\end{array}\right] \tau,
$$

where $a_{1}=m_{p} l_{c_{1}}^{2}+m_{w} l^{2}+I_{p}+I_{w}, a_{2}=I_{w}, a_{3}=g\left(m_{p} l_{c_{1}}+m_{w} l\right)$ and the control input $u=\tau$ is the motor torque. The remaining parameters are listed in Table 1. The dynamic model of the IWP can be written in PCH form (1) with $n=2, m=1, G=e_{2}=\left[\begin{array}{ll}0 & 1\end{array}\right]^{\top}$ and

$$
M=\left[\begin{array}{cc}
a_{1}+a_{2} & a_{2} \\
a_{2} & a_{2}
\end{array}\right], \quad V\left(q_{1}\right)=a_{3}\left(\cos \left(q_{1}\right)+1\right) \text {. }
$$


Table 1

The parameters of the inertia wheel pendulum

\begin{tabular}{|c|c|c|l|}
\hline Symb & Description & Value & Unit \\
\hline$m_{p}$ & Mass of the pendulum & 0.2164 & $\mathrm{~kg}$ \\
\hline$l$ & Total length of the pendulum & 0.2346 & $\mathrm{~m}$ \\
\hline$l_{c_{1}}$ & length to the pendulum center of mass & 0.1173 & $\mathrm{~m}$ \\
\hline$I_{p}$ & Moment of inertia of the pendulum & $2.233 \times 10^{-4}$ & $\mathrm{~kg} \cdot \mathrm{m}^{2}$ \\
\hline$m_{w}$ & Mass of the wheel & 0.0850 & $\mathrm{~kg}$ \\
\hline$I_{w}$ & Moment of inertia of the wheel & $2.495 \times 10^{-5}$ & $\mathrm{~kg} \cdot \mathrm{m}^{2}$ \\
\hline$g$ & Gravitational acceleration & 9.81 & $\mathrm{~m} / \mathrm{s}^{2}$ \\
\hline
\end{tabular}

\subsection{Controller design}

We apply Proposition 3.1 to design a controller for the IWP, to swing up the pendulum by spinning the wheel and to asymptotically stabilize it at its upward position $q_{e}=\left(0, q_{2}\right)$ for any $q_{2} \in[0,2 \pi]$. First the energy shaping controller $u_{e s}$ is designed, and then some damping is added to the closed-loop system by designing the damping injection controller $u_{d i}$.

\section{Reshaping the total energy}

Following the procedures in Proposition 3.1, we fix $M_{d}$ in the form of

$$
M_{d}=\Delta\left[\begin{array}{cc}
m_{1} & \frac{m_{1} a_{2}}{a_{1}+a_{2}}+\varepsilon \\
\frac{m_{1} a_{2}}{a_{1}+a_{2}}+\varepsilon & m_{3}
\end{array}\right]
$$

where $\Delta=a_{1} a_{2}$. With this choice of $M_{d}$, and having $G^{\perp}=\left[\begin{array}{ll}1 & 0\end{array}\right]$, the PDE (9) becomes

$$
\left[\begin{array}{ll}
1 & 0
\end{array}\right]\left\{\left[\begin{array}{c}
-a_{3} \sin \left(q_{1}\right) \\
0
\end{array}\right]-\Delta\left[\begin{array}{cc}
m_{1} & \frac{m_{1} a_{2}}{a_{1}+a_{2}}+\varepsilon \\
\frac{m_{1} a_{2}}{a_{1}+a_{2}}+\varepsilon & m_{3}
\end{array}\right]\left[\begin{array}{cc}
\frac{a_{2}}{\Delta} & \frac{-a_{2}}{\Delta} \\
\frac{-a_{2}}{\Delta} & \frac{a_{1}+a_{2}}{\Delta}
\end{array}\right]\left[\begin{array}{c}
\nabla_{q_{1}} V_{d} \\
\nabla_{q_{2}} V_{d}
\end{array}\right]\right\}=0,
$$

which further gives

$$
\left(m_{1} a_{2}-\frac{m_{1} a_{2}^{2}}{a_{1}+a_{2}}-\varepsilon a_{2}\right) \nabla_{q_{1}} V_{d}+\varepsilon\left(a_{1}+a_{2}\right) \nabla_{q_{2}} V_{d}=-a_{3} \sin \left(q_{1}\right) .
$$

Solving this PDE produces the desired potential energy

$$
\begin{aligned}
V_{d}(q) & =-a_{3} \gamma_{1} \cos \left(q_{1}\right)+\Psi(x(q)), \\
x(q) & =q_{2}+\gamma_{1} \varepsilon\left(a_{1}+a_{2}\right) q_{1}
\end{aligned}
$$


with $\gamma_{1}=\frac{a_{1}+a_{2}}{a_{2}\left(\varepsilon\left(a_{1}+a_{2}\right)-a_{1} m_{1}\right)}>0$. The function $\Psi(\cdot)$ in $(29)$ is an arbitrary differentiable function that must be chosen to satisfy condition (6). This condition, along with the conditions $m_{1}>0$ and $m_{1} m_{3}>m_{2}^{2}$ are satisfied by choosing $\Psi(q)=\frac{1}{2} K_{p}(x(q))^{2}$, where $K_{p}>0$ is the gain of the energy shaping controller. Substituting all the terms into (10), we obtain the energy shaping controller

$$
u_{e s}=\gamma_{2} \sin \left(q_{1}\right)+K_{p} \gamma_{3}\left(q_{2}+\gamma_{1} \varepsilon\left(a_{1}+a_{2}\right) q_{1}\right) \text {, }
$$

with $\gamma_{2}=-m_{0} \gamma_{1} a_{2}\left(m_{2}-m_{3}\right)$ and $\gamma_{3}=m_{2} a_{2}\left(\gamma_{1}-1\right)+m_{3}\left(a_{1}+a_{2}\right)\left(1-\gamma_{1} a_{2}\right)$.

\section{Damping assignment}

The damping injection controller follows the construction (11). Given $M_{d}$ that has been obtained when reshaping the total energy, we have

$$
\begin{aligned}
\nabla_{p} H_{d}=M_{d}^{-1} p & =\frac{\Delta}{\Delta_{d}}\left[\begin{array}{cc}
m_{3} & -\frac{m_{1} a_{2}}{a_{1}+a_{2}}-\varepsilon \\
-\frac{m_{1} a_{2}}{a_{1}+a_{2}}-\varepsilon & m_{1}
\end{array}\right]\left[\begin{array}{l}
p_{1} \\
p_{2}
\end{array}\right] \\
& =\frac{\Delta}{\Delta_{d}}\left[\begin{array}{c}
m_{3} p_{1}-\left(\frac{m_{1} a_{2}}{a_{1}+a_{2}}+\varepsilon\right) p_{2} \\
-\left(\frac{m_{1} a_{2}}{a_{1}+a_{2}}+\varepsilon\right) p_{1}+m_{1} p_{2}
\end{array}\right]
\end{aligned}
$$

with $\Delta_{d}=\operatorname{det}\left(M_{d}\right)=\Delta^{2}\left(m_{1} m_{3}-m_{2}^{2}\right)$. Substituting (32) into (11), the damping injection controller is then

$$
u_{d i}=-K_{v} \frac{\Delta}{\Delta_{d}}\left(-\left(\frac{m_{1} a_{2}}{a_{1}+a_{2}}+\varepsilon\right) p_{1}+m_{1} p_{2}\right) .
$$

We can conclude the IDA-PBC design for the IWP by the following corollary.

Corollary 4.1 The state feedback controller (31), (33) with $K_{p}>0, K_{v}>0$ and $m_{3}>\frac{m_{2}^{2}}{m_{1}}$, is an asymptotically stabilizing controller for the IWP (27) at its unstable equilibrium point $q=\left(0, q_{2}\right)$ for any $q_{2} \in[0,2 \pi]$.

Proof of Corollary 4.1: The proof can be established by verifying that $V_{d}$ satisfies Conditions 2.1 and 2.2, and $M_{d}=M_{d}^{\top}$ is positive definite. Thus, $H_{d}$ qualifies as a Lyapunov function. Moreover, asymptotic stability is proved invoking LaSalle's invariance principle (see the proof of Corollary 5.1).

\subsection{Simulation results}

Some simulation results are obtained by applying the controller (31), (33) to the inertia wheel pendulum model. In all simulations, the initial condition $\left[q_{0}, p_{0}\right]=[\pi, 0,0,0.05]$, i.e. the pendulum vertical downward position, is used. The parameters and gains for the stabilizing IDA-PBC controller are $m_{1}=0.7$, $m_{3}=3.48$ and $\epsilon=1$. 
Figure 2 shows the response for different values of $K_{p}$, with $K_{v}=2 \times 10^{-5}$. Their corresponding control inputs are shown in Figure 3. As shown, the pendulum asymptotically converges toward its upward position, from its downward position, i.e. almost global stabilization. Observe that the states converge faster for small $K_{p}$, while high-gain controller causes more oscillations.
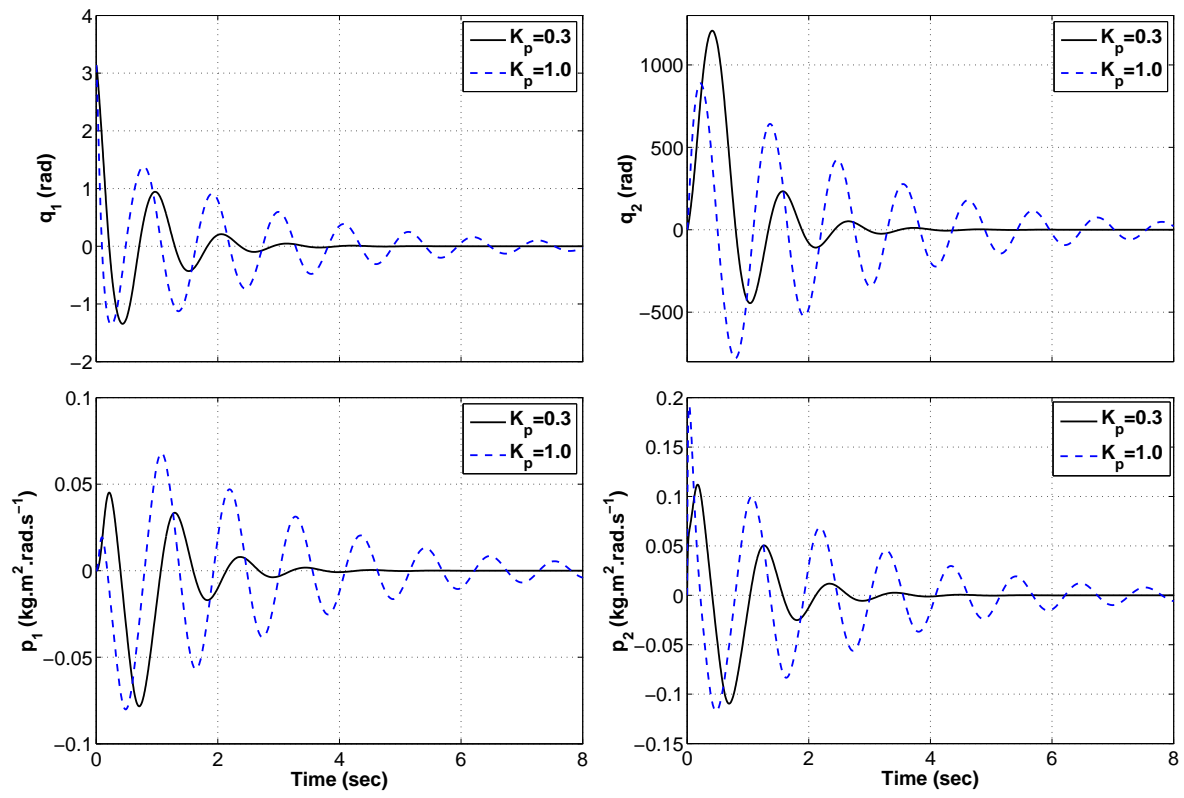

Fig. 2. Trajectories of the IWP for different values of $K_{p}$.

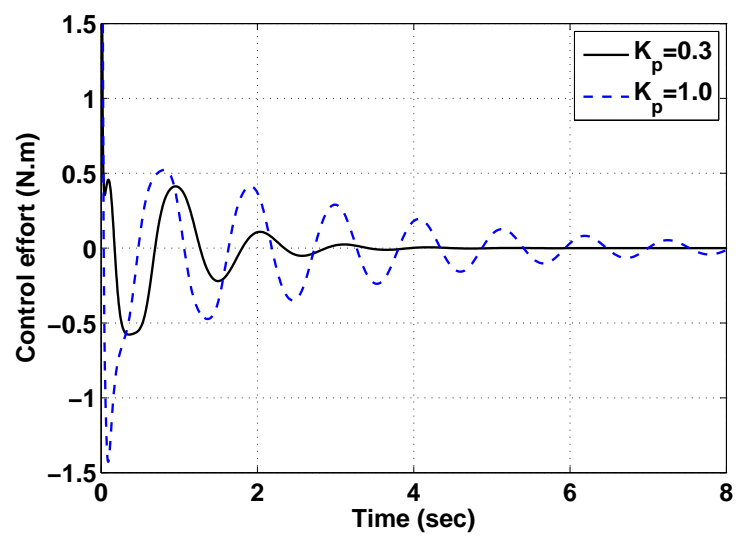

Fig. 3. Control torque of the IWP for different values of $K_{p}$.

Figures 4 and 5 illustrate the effect of varying the damping gain $K_{v}$ for a constant $K_{p}=0.3$. As expected, increasing this damping gain leads to achieving faster convergence with less oscillations.

\section{Non-Separable Hamiltonian Systems: the Rotary Inverted Pen- dulum Example}

In this section, we illustrate the effectiveness of our proposed method applied to a more complex structure of UMSs, the non-separable systems, using a 

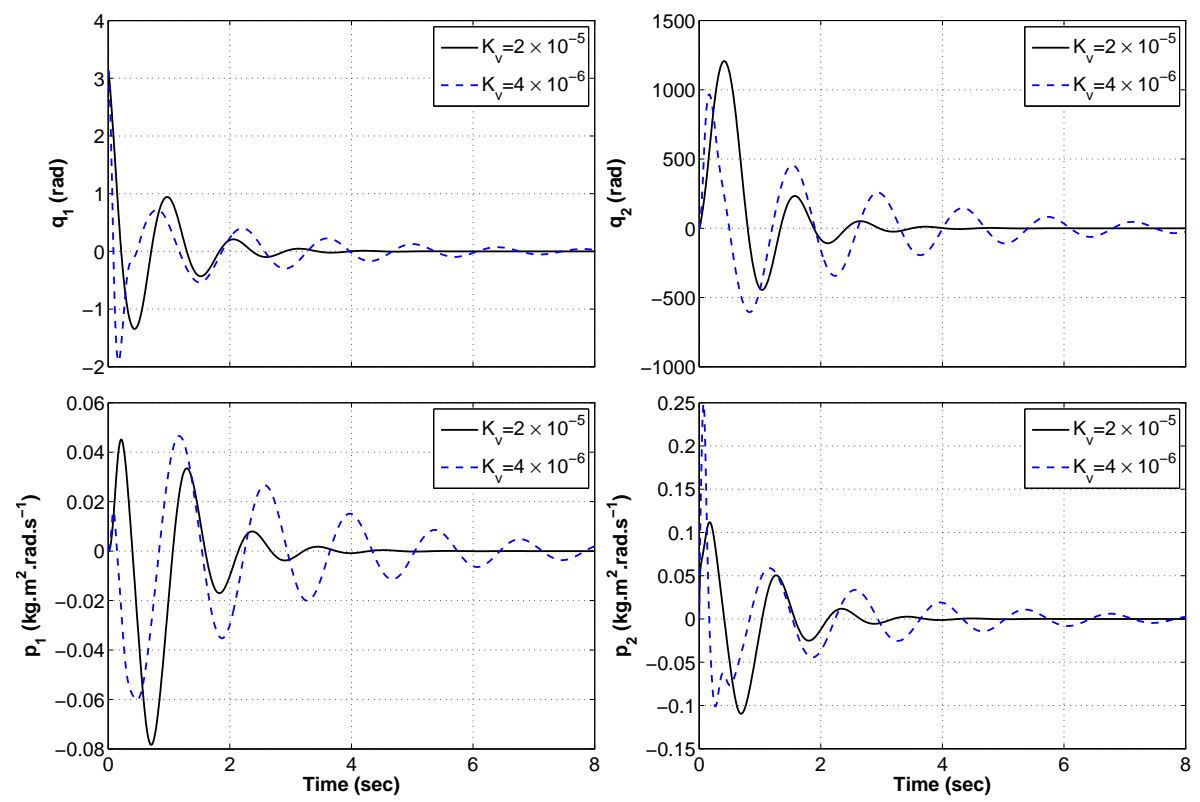

Fig. 4. Trajectories of the IWP for different values of $K_{v}$.

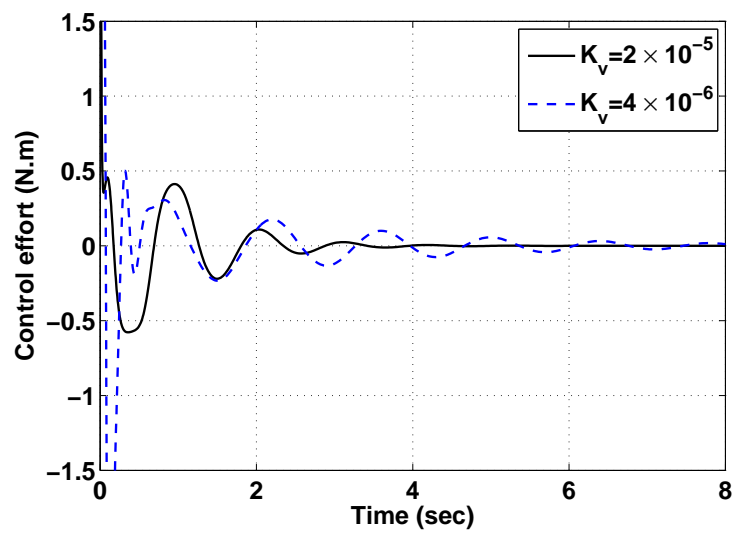

Fig. 5. Control torque of the IWP for different values of $K_{v}$.

rotary inverted pendulum. We show that this technique reduces the design complexity, while at the same time preserves the effectiveness of the IDAPBC to asymptotically stabilize the pendulum at its upright position.

The control of a rotary inverted pendulum has been classically approached via switching between two controllers. The first controller swings the pendulum up close to its upright position from its downward position, and usually designed using energy based techniques [5]. At this point, the second, a balancing controller -often a linear controller- is applied to stabilize the pendulum at the desired upright position. Some energy based methods to control this system have been proposed in literature such as a swing-up control law for general pendulum-like systems [5], energy-based controller involving passivity to enforce the system to converge to its homoclinic orbit [13], and a strategy based on controlled Lagrangian framework [21], to mention a few. 
Two works have been reported for the control of this system within the PCH structure; in [4], a method which incorporates shaping the 'normalized' Hamiltonian function of the system and energy damping/pumping has been developed. In [38], the IDA-PBC method is adopted, and the simplification of the kinetic energy PDEs has been achieved using quasi-linearization. In this example, we apply Proposition 3.2, exploiting the full nonlinear dynamics of the rotary inverted pendulum.

\subsection{Rotary inverted pendulum model}

We use the Quanser QUBE-Servo inverted pendulum module, as shown in Figure 6 together with the simplified free body diagram of its mechanical part. This system consists of an inverted pendulum which is attached at the end of a motor-driven horizontally-rotating arm. The pendulum is also free to rotate in a vertical plane. Thus, the system has 2-DOF: the angular position of the $\operatorname{arm}(\alpha)$ and the angular position of the pendulum $(\theta)$. This system is underactuated because only the arm is subjected to an input torque (applied by a DC motor). The parameters along with their physical values are listed in Table 2. The equations of motion of the system can be derived from the
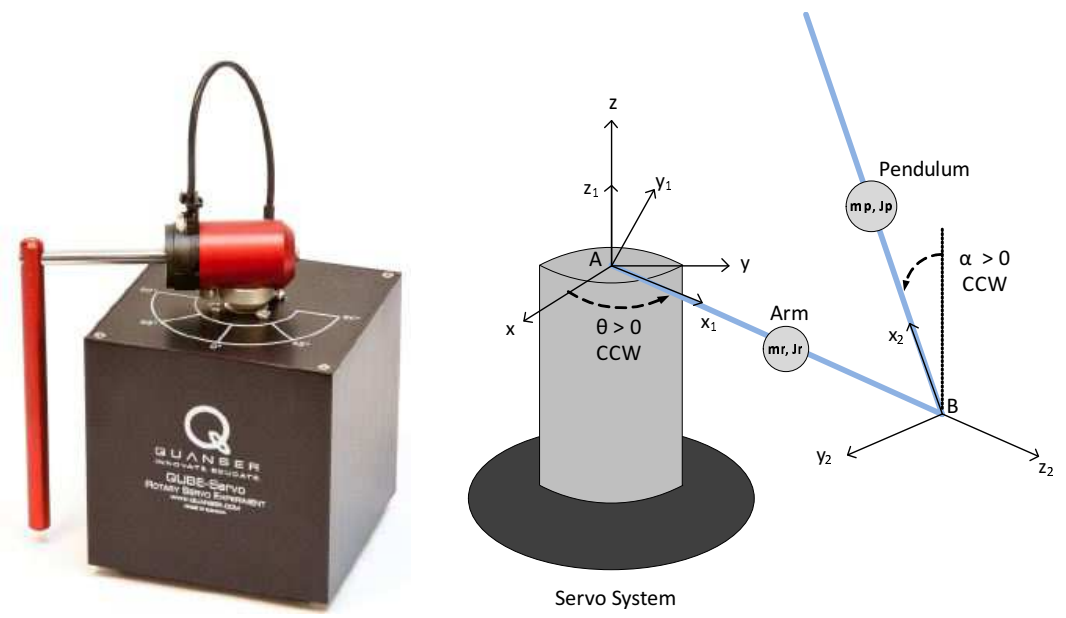

Fig. 6. Quanser QUBE-Servo inverted pendulum system [27].

standard Euler-Lagrange method as [27]:

$$
\begin{aligned}
& -\frac{1}{2} m_{p} L_{p} L_{r} \cos (\alpha) \ddot{\theta}+\left(J_{p}+\frac{1}{4} m_{p} L_{p}^{2}\right) \ddot{\alpha}-\frac{1}{2} m_{p} L_{p} g \sin (\alpha) \\
& -\frac{1}{4} m_{p} L_{p}^{2} \cos (\alpha) \sin (\alpha) \dot{\theta}^{2}=-B_{p} \dot{\alpha} \\
& \left(J_{r}+m_{p} L_{r}^{2}+\frac{1}{4} m_{r} L_{r}^{2}+\left(J_{p}+\frac{1}{4} m_{p} L_{p}^{2}\right) \sin ^{2}(\alpha)\right) \ddot{\theta}-\frac{1}{2} m_{p} L_{p} L_{r} \cos (\alpha) \ddot{\alpha} \\
& +\frac{1}{2} m_{p} L_{p} L_{r} \sin (\alpha) \dot{\alpha}^{2}+\frac{1}{2} m_{p} L_{p}^{2} \cos (\alpha) \sin (\alpha) \dot{\theta} \dot{\alpha}=\tau-B_{r} \dot{\theta}
\end{aligned}
$$


To apply the IDA-PBC design, we need to obtain the $\mathrm{PCH}$ representation of the system. We define the generalized coordinate $q$ to be $q=\left[\begin{array}{ll}q_{1} & q_{2}\end{array}\right]^{\top}=\left[\begin{array}{ll}\alpha \theta & \theta\end{array}\right]^{\top}$ and introduce the shorten notations for the parameters

$\gamma=J_{p}+\frac{1}{4} m_{p} L_{p}^{2}, \quad \rho=J_{r}+m_{p} L_{r}^{2}+\frac{1}{4} m_{r} L_{r}^{2}, \quad \sigma=\frac{1}{2} m_{p} L_{p} L_{r}, \quad \kappa=\frac{1}{2} m_{p} g L_{p}$.

Applying Newton's Second Law for rotational motion while ignoring the effect of friction, from (34)-(35) we extract the inertia matrix

$$
M(q)=\left[\begin{array}{cc}
\gamma & -\sigma \cos \left(q_{1}\right) \\
-\sigma \cos \left(q_{1}\right) & \rho+\gamma \sin ^{2}\left(q_{1}\right)
\end{array}\right],
$$

and also the potential energy of the system

$$
V\left(q_{1}\right)=\kappa\left(1+\cos \left(q_{1}\right)\right) .
$$

The Hamiltonian function of the system can then be obtained using (2). Moreover, the PCH model of the rotary pendulum can be described by (1) with $u=\tau$, and the input matrix $G=e_{2}=\left[\begin{array}{ll}0 & 1\end{array}\right]^{\top}$.

Table 2

The parameters of the Quanser QUBE-Servo inverted pendulum

\begin{tabular}{|c|l|l|l|}
\hline Symbol & Description & Value & Unit \\
\hline$m_{p}$ & Mass of pendulum & 0.024 & $\mathrm{~kg}$ \\
\hline$L_{p}$ & Total length of pendulum & 0.129 & $\mathrm{~m}$ \\
\hline$J_{p}$ & Moment of inertia of pendulum & $3.33 \times 10^{-5}$ & $\mathrm{~kg} \cdot \mathrm{m}^{2}$ \\
\hline$m_{r}$ & Mass of arm & 0.095 & $\mathrm{~kg}$ \\
\hline$L_{r}$ & Total length of arm & 0.085 & $\mathrm{~m}$ \\
\hline$J_{r}$ & Moment of inertia of arm & $5.72 \times 10^{-5}$ & $\mathrm{~kg} \cdot \mathrm{m}^{2}$ \\
\hline$v$ & Output Voltage range & \pm 10 & volt \\
\hline
\end{tabular}

\subsection{Controller design}

We apply the procedure given in Section 3, in particular Proposition 3.2, to design the controller for the system. The main objective is to asymptotically stabilize the rotary inverted pendulum at its unstable equilibrium point $q_{e}=$ $\left(0, q_{2}\right)$ for any $q_{2} \in[0,2 \pi]$. First we design the energy shaping controller $u_{e s}$ and then adding the damping to the closed-loop system by designing the damping injection controller $u_{d i}$. 


\section{Reshaping the total energy}

We start with parametrizing the inertia matrix $M_{d}$, then solve the PDE of the potential energy. From (36), we obtain the inverse inertia matrix

$$
M^{-1}(q)=\frac{1}{\Delta}\left[\begin{array}{cc}
\rho+\gamma \sin ^{2}\left(q_{1}\right) & \sigma \cos \left(q_{1}\right) \\
\sigma \cos \left(q_{1}\right) & \gamma
\end{array}\right],
$$

where $\Delta=\operatorname{det}(M)=\gamma \rho+\gamma^{2} \sin ^{2}\left(q_{1}\right)-\sigma^{2} \cos ^{2}\left(q_{1}\right)$. Because $M$ depends on $q_{1}$, it is clear that $M^{-1}$ is a complicated matrix. Solving directly the PDEs (8)-(9) will require also tedious computations, that will lead to an unreasonable form of the controller.

Applying Proposition 3.2, with $G^{\perp}=[10]$, the potential energy PDE (9)

$$
\begin{aligned}
& {\left[\begin{array}{ll}
1 & 0
\end{array}\right]\left\{\left[\begin{array}{c}
-\kappa \sin \left(q_{1}\right) \\
0
\end{array}\right]-\Delta\left[\begin{array}{l}
m_{1}\left(q_{1}\right) m_{2}\left(q_{1}\right) \\
m_{2}\left(q_{1}\right) \\
m_{3}\left(q_{1}\right)
\end{array}\right]\right.} \\
& \left.\times \frac{1}{\Delta}\left[\begin{array}{cc}
\rho+\gamma \sin ^{2}\left(q_{1}\right) & \sigma \cos \left(q_{1}\right) \\
\sigma \cos \left(q_{1}\right) & \gamma
\end{array}\right]\left[\begin{array}{c}
\nabla_{q_{1}} V_{d} \\
\nabla_{q_{2}} V_{d}
\end{array}\right]\right\}=0,
\end{aligned}
$$

can be written in its simplest form

$$
\nabla_{q_{1}} V_{d}=-\frac{\gamma \kappa \sin \left(q_{1}\right)}{m_{1} \Delta},
$$

by choosing $m_{2}=\frac{k_{2}}{k_{1}} m_{1}$, and including $\Delta=\operatorname{det}(M)=\gamma \rho+\gamma^{2} \sin ^{2}\left(q_{1}\right)-$ $\sigma^{2} \cos ^{2}\left(q_{1}\right)$ in the expression of $M_{d}$. The solution for the PDE (39) is given by

$$
V_{d}(q)=-\gamma \kappa \int_{0}^{q_{1}} \frac{\sin (x)}{m_{1}(x) \Delta(x)} d x+\Psi\left(q_{2}\right),
$$

where, the function $\Psi(\cdot)$ is an arbitrary differentiable function that must be chosen to satisfy (6). The second step is to fix $m_{1}$ in (39) such that the solution of this PDE satisfies Conditions 2.1 and 2.2. Notice that $M$ is a function of $q_{1}$ only, hence, we can simply take $M_{d}$ as a function of $q_{1}$ too. Among the possible choices, we have fixed the desired inertia matrix as

$$
M_{d}(q)=\Delta\left[\begin{array}{cc}
\left(\cos \left(q_{1}\right)+\epsilon\right) & \frac{-\sigma \cos \left(q_{1}\right)\left(\cos \left(q_{1}\right)+\epsilon\right)}{\gamma} \\
\frac{-\sigma \cos \left(q_{1}\right)\left(\cos \left(q_{1}\right)+\epsilon\right)}{\gamma} & m_{3}
\end{array}\right],
$$

choosing $\epsilon>\max \left|\cos \left(q_{1}\right)\right|=1$ to guarantee $m_{1}>0$, and hence $M_{d}>0 \forall q_{1} \epsilon$ $[0,2 \pi]$. Then, the desired potential energy function $V_{d}$ is computed (using 
Maple software) as

$$
V_{d}(q)=\lambda_{1}\left(-\lambda_{2} \tanh ^{-1}\left(\lambda_{2} \cos \left(q_{1}\right)\right)+\ln \left(\cos \left(q_{1}\right)+\epsilon\right)\right)+\Psi\left(q_{2}\right)
$$

with

$$
\lambda_{1}=\frac{\kappa \gamma}{\gamma \rho+\gamma^{2}-\sigma^{2} \epsilon^{2}-\gamma^{2} \epsilon^{2}}>0, \quad \lambda_{2}=\sqrt{\frac{\gamma^{2}+\sigma^{2}}{\gamma(\rho+\gamma)}}, \quad\left(0<\lambda_{2}<1\right) .
$$

The function $\Psi(\cdot)$ is an arbitrary differentiable function that must be chosen to satisfy condition (6). This condition, along with the conditions $m_{1}>0$ and $m_{1} m_{3}>m_{2}^{2}$ are satisfied by choosing $\Psi\left(q_{2}\right)=\frac{1}{2} K_{p} q_{2}^{2}$, where $K_{p}>0$ is the gain of energy shaping controller.

Remark 5.1 For this particular design, we have fixed $m_{1}=\cos \left(q_{1}\right)+\epsilon$. The term $\cos \left(q_{1}\right)$ ensures that $V_{d}$ is minimum at $q_{e}$, and $\epsilon$ is added to guarantee the positive definiteness of $M_{d}$ in the whole DoA.

Now, using (26) we compute $J_{2}$, which after lengthy but straightforward calculations is obtained as

$$
J_{2}=\left[\begin{array}{cc}
0 & j_{2} \\
-j_{2} & 0
\end{array}\right]
$$

where,

$$
j_{2}=\frac{K_{j} \gamma \Delta_{d}}{2 \Delta} \frac{\left(\left(\varphi \mathcal{B}_{1}-\mathcal{A}_{1}\right) p_{1}^{2}+2\left(\varphi \mathcal{B}_{2}-\mathcal{A}_{2}\right) p_{1} p_{2}+\left(\varphi \mathcal{B}_{3}-\mathcal{A}_{3}\right) p_{2}^{2}\right)}{\left(\cos \left(q_{1}\right)+\epsilon\right)\left(\sigma \cos \left(q_{1}\right) p_{1}+\gamma p_{2}\right)}
$$

with $K_{j}>0$, and

$$
\begin{gathered}
\mathcal{A}_{1}=\frac{\sin \left(2 q_{1}\right)}{\Delta^{2}}\left(\gamma \Delta-\left(\rho+\gamma \sin ^{2}\left(q_{1}\right)\right)\left(\gamma^{2}+\sigma^{2}\right)\right) \\
\mathcal{A}_{2}=-\frac{\sigma \sin \left(q_{1}\right)}{\Delta^{2}}\left(\Delta+2 \cos ^{2}\left(q_{1}\right)\left(\gamma^{2}+\sigma^{2}\right)\right) \\
\mathcal{A}_{3}=-\frac{\gamma\left(\gamma^{2}+\sigma^{2}\right) \sin \left(2 q_{1}\right)}{\Delta^{2}}, \\
\mathcal{B}_{1}=\frac{m_{3} \sin \left(q_{1}\right)}{\left(\gamma \Delta_{d}\right)^{2}}\left(-2 \gamma^{2} \Delta_{d} \cos \left(q_{1}\right)\left(\gamma^{2}+\sigma^{2}\right)+\Delta^{3}\left(m_{3} \gamma^{2}-\epsilon \sigma^{2} \cos ^{2}\left(q_{1}\right)-\sigma^{2} \cos ^{3}\left(q_{1}\right)\right)\right. \\
\left.-\sigma^{2} \Delta^{3} \cos \left(q_{1}\right)\left(\epsilon+\cos \left(q_{1}\right)\right)\left(2 \epsilon+3 \cos \left(q_{1}\right)\right)\right) \\
\mathcal{B}_{2}=-\frac{\sigma \sin \left(q_{1}\right)\left(\epsilon+\cos \left(q_{1}\right)\right)}{\gamma \Delta_{d}\left(m_{3} \gamma^{2}-\epsilon \sigma^{2} \cos ^{2}\left(q_{1}\right)-\sigma^{2} \cos ^{3}\left(q_{1}\right)\right)}\left(\sigma^{2} \Delta \cos ^{2}\left(q_{1}\right)\left(2 \epsilon+3 \cos \left(q_{1}\right)\right)\right. \\
\left.+\left(2 \cos ^{2}\left(q_{1}\right)\left(\gamma^{2}+\sigma^{2}\right)+\Delta\right)\left(m_{3} \gamma^{2}-\epsilon \sigma^{2} \cos ^{2}\left(q_{1}\right)-\sigma^{2} \cos ^{3}\left(q_{1}\right)\right)\right),
\end{gathered}
$$




$$
\begin{aligned}
\mathcal{B}_{3}= & -\frac{\sin \left(q_{1}\right) \cos \left(q_{1}\right)\left(\epsilon+\cos \left(q_{1}\right)\right)}{\Delta_{d}\left(m_{3} \gamma^{2}-\epsilon \sigma^{2} \cos ^{2}\left(q_{1}\right)-\sigma^{2} \cos ^{3}\left(q_{1}\right)\right)} \times \\
& \left(2\left(\gamma^{2}+\sigma^{2}\right)\left(m_{3} \gamma^{2}-\epsilon \sigma^{2} \cos ^{2}\left(q_{1}\right)-\sigma^{2} \cos ^{3}\left(q_{1}\right)\right)+\sigma^{2} \Delta\left(2 \epsilon+3 \cos \left(q_{1}\right)\right)\right), \\
\varphi= & \left(\cos \left(q_{1}\right)+\epsilon\right)\left(\rho+\gamma \sin ^{2}\left(q_{1}\right)-\frac{\sigma^{2} \cos ^{2}\left(q_{1}\right)}{\gamma}\right)
\end{aligned}
$$

and $\Delta_{d}=\operatorname{det}\left(M_{d}\right)=\frac{\Delta^{2}}{\gamma^{2}}\left(\cos \left(q_{1}\right)+\epsilon\right)\left(m_{3} \gamma^{2}-\sigma^{2} \cos ^{2}\left(q_{1}\right)\left(\cos \left(q_{1}\right)+\epsilon\right)\right)$. Substituting all terms into (10), the energy shaping controller is obtained as

$$
\begin{aligned}
u_{e s}= & -\frac{\sigma \cos \left(q_{1}\right)}{\gamma}\left(\gamma m_{3}-\left(\rho+\gamma \sin ^{2}\left(q_{1}\right)\right)\left(\epsilon+\cos \left(q_{1}\right)\right)\right)\left(\frac{\mathcal{B}_{1}}{2} p_{1}^{2}+\mathcal{B}_{2} p_{1} p_{2}+\frac{\mathcal{B}_{3}}{2} p_{2}^{2}\right. \\
& \left.+\frac{\epsilon \lambda_{1} \lambda_{2}^{2} \sin \left(q_{1}\right)}{1-\lambda_{2}^{2} \cos ^{2}\left(q_{1}\right)}-\frac{\lambda_{1} \sin \left(q_{1}\right)}{\epsilon+\cos \left(q_{1}\right)}\right)-\left(\gamma m_{3}-\frac{\sigma^{2} \cos ^{2}\left(q_{1}\right)\left(\cos \left(q_{1}\right)+\epsilon\right)}{\gamma}\right) K_{p} q_{2} \\
& -j_{2} \frac{\Delta}{\gamma \Delta_{d}}\left(\gamma m_{3} p_{1}+\sigma \cos \left(q_{1}\right)\left(\cos \left(q_{1}\right)+\epsilon\right) p_{2}\right)
\end{aligned}
$$

\section{Damping assignment}

The damping injection controller follows the construction (11). Given $M_{d}$ that has been obtained when reshaping the total energy, we have

$$
\begin{aligned}
\nabla_{p} H_{d}=M_{d}^{-1} p & =\frac{\Delta}{\Delta_{d}}\left[\begin{array}{cc}
m_{3} & \frac{\sigma \cos \left(q_{1}\right)\left(\cos \left(q_{1}\right)+\epsilon\right)}{\gamma} \\
\frac{\sigma \cos \left(q_{1}\right)\left(\cos \left(q_{1}\right)+\epsilon\right)}{\gamma} & \epsilon+\cos \left(q_{1}\right)
\end{array}\right]\left[\begin{array}{l}
p_{1} \\
p_{2}
\end{array}\right] \\
& =\frac{\Delta}{\Delta_{d}}\left[\begin{array}{c}
m_{3} p_{1}+\frac{\sigma \cos \left(q_{1}\right)\left(\cos \left(q_{1}\right)+\epsilon\right)}{\gamma} p_{2} \\
\frac{\sigma \cos \left(q_{1}\right)\left(\cos \left(q_{1}\right)+\epsilon\right)}{\gamma} p_{1}+\left(\cos \left(q_{1}\right)+\epsilon\right) p_{2}
\end{array}\right]
\end{aligned}
$$

Substituting (46) into (11), the damping injection controller is obtained as

$$
u_{d i}=-\frac{K_{v} \Delta\left(\cos \left(q_{1}\right)+\epsilon\right)}{\gamma \Delta_{d}}\left(\sigma \cos \left(q_{1}\right) p_{1}+\gamma p_{2}\right) .
$$

Now, we can conclude the IDA-PBC controller design for our rotary inverted pendulum by stating the following corollary.

Corollary 5.1 The state feedback controller (45), (47), with $m_{3}>\left(\cos \left(q_{1}\right)+\right.$ $\epsilon) \frac{\sigma^{2} \cos ^{2}\left(q_{1}\right)}{\gamma^{2}}, \epsilon>1$ and $K_{p}, K_{v}, K_{j}>0$ is an asymptotically stabilizing controller for the rotary pendulum system (34)-(35) at its unstable equilibrium point $q=\left(0, q_{2}\right)$ for any $q_{2} \in[0,2 \pi]$.

Proof of Corollary 5.1: The proof of Corollary 5.1 is discussed next in Subsection 5.3. 


\subsection{Stability analysis}

As mentioned earlier (see also Proposition 1 in [2]), one important property of the IDA-PBC method is that the closed-loop energy function $H_{d}(q, p)$ qualifies as a Lyapunov function candidate $W(q, p)$, thus has a stable equilibrium point at $\left(q_{e}, 0\right)$. For this to apply, $H_{d}(q, p)$ itself must satisfy two conditions: 1) $M_{d}$ is positive definite and symmetric, and 2) the closed-loop potential energy function $V_{d}$ must have an isolated minimum at this equilibrium. Moreover, this equilibrium is asymptotically stable provided that the closed-loop system satisfies the detectability condition from the output $y_{d}=G^{\top} \nabla_{p} H_{d}$.

As explained through the design procedures, a sufficient condition to guarantee the positive definiteness of $M_{d}$ in (41) is that $m_{1}>0$ and $m_{1} m_{3}>m_{2}^{2}$. The earlier is achieved by assigning $m_{1}=\left(\cos \left(q_{1}\right)+\epsilon\right)$ with $\epsilon>\left|\cos \left(q_{1}\right)\right|$. By choosing $m_{3}$ such that it satisfies the condition $m_{3}>\left(\epsilon+\cos \left(q_{1}\right)\right) \frac{\sigma^{2} \cos ^{2}\left(q_{1}\right)}{\gamma^{2}}$, for instance $m_{3}:=\left(\cos \left(q_{1}\right)+\epsilon\right) \frac{\sigma^{2} \cos ^{2}\left(q_{1}\right)}{\gamma^{2}}+\mu$ with a constant $\mu>0$, the latter condition is also achieved. Finally, it is clear that $M_{d}$ is symmetric since the elements $m_{12}=m_{21}:=m_{2}$.

To prove the assignment of the minimum of the potential energy

$$
q_{e}=\arg \min H_{d}(q)=\arg \min V_{d}(q),
$$

we will show that Conditions 2.1 and 2.2 are satisfied. The gradient of $V_{d}$ is

$$
\nabla_{q} V_{d}=\lambda_{1} \sin \left(q_{1}\right)\left[\begin{array}{c}
\frac{\epsilon\left(\sigma^{2}+\gamma^{2}\right)}{\gamma(\rho+\gamma)-\left(\sigma^{2}+\gamma^{2}\right) \cos ^{2}\left(q_{1}\right)}-\frac{1}{\left(\epsilon+\cos \left(q_{1}\right)\right)} \\
K_{p} q_{2}
\end{array}\right]
$$

Solving at $q_{e}=(0,0)$ yields $\left.\nabla_{q} V_{d}\right|_{q_{e}}=\left[\begin{array}{ll}0 & 0\end{array}\right]^{\top}$. Hence, Condition 2.1 is satisfied. To verify that the Hessian of $V_{d}$ is positive definite, we evaluate

$$
\nabla_{q}^{2} V_{d}=\operatorname{diag}\left[\left(\nabla_{q}^{2} V_{d}\right)_{11}, K_{p}\right]
$$

where

$$
\begin{aligned}
\left(\nabla_{q}^{2} V_{d}\right)_{11}= & \left(\frac{\lambda_{1} \cos \left(q_{1}\right) \epsilon\left(\sigma^{2}+\gamma^{2}\right)}{\gamma(\rho+\gamma)-\left(\sigma^{2}+\gamma^{2}\right) \cos ^{2}\left(q_{1}\right)}-\frac{\lambda_{1} \cos \left(q_{1}\right)}{\left(\epsilon+\cos \left(q_{1}\right)\right)}\right) \\
& -\left(\frac{2 \epsilon \cos \left(q_{1}\right)\left(\sigma^{2}+\gamma^{2}\right)^{2} \lambda_{1} \sin ^{2}\left(q_{1}\right)}{\left(\gamma(\rho+\gamma)-\left(\sigma^{2}+\gamma^{2}\right) \cos ^{2}\left(q_{1}\right)\right)^{2}}+\frac{\lambda_{1} \sin ^{2}\left(q_{1}\right)}{\left(\epsilon+\cos \left(q_{1}\right)\right)^{2}}\right)
\end{aligned}
$$

at $q_{e}$, thus we obtain

$$
\left.\nabla_{q}^{2} V_{d}\right|_{q_{e}}=\operatorname{diag}\left[\lambda_{1}\left(\frac{\epsilon\left(\sigma^{2}+\gamma^{2}\right)}{\gamma \rho-\sigma^{2}}-\frac{1}{(\epsilon+1)}\right), K_{p}\right],
$$


which is positive definite for $K_{p}, \lambda_{1}>0$ and $\epsilon>1$.

Notice that $\epsilon>1$ in (49) guarantees that $\frac{\epsilon\left(\sigma^{2}+\gamma^{2}\right)}{\gamma \rho-\sigma^{2}}>\frac{1}{(\epsilon+1)}$. Hence Condition 2.2 also holds. Since all conditions are satisfied, we can conclude that $H_{d}$ qualifies as a Lyapunov function, i.e.

$$
H_{d}(q, p)=W(q, p)=\frac{1}{2} p^{\top} M_{d}^{-1}(q) p+V_{d}(q)
$$

and so that the rotary inverted pendulum system (34)-(35) is stabilized at its unstable equilibrium point $q=\left(0, q_{2}\right)$ by the proposed IDA-PBC design. To prove that the system is asymptotically stable, either detectability condition should be guaranteed, or, since the closed-loop energy function of the system qualifies as a Lyapunov function candidate, we can invoke LaSalle's invariance principle as follows.

The derivative of (50) along the trajectories of the closed-loop system is

$$
\begin{aligned}
\dot{W}(q, p) & =\left(\nabla_{q} H_{d}\right)^{\top} \dot{q}+\left(\nabla_{p} H_{d}\right)^{\top} \dot{p} \\
& =-p^{\top} M_{d}^{-1} G K_{v} G^{\top} M_{d}^{-1} p \\
& =-K_{v}\left(G^{\top} \nabla_{p} H_{d}\right)^{2} \leq 0,
\end{aligned}
$$

where $K_{v}>0$. Thus, $\dot{W}$ is negative semidefinite. Using LaSalle's invariance principle [15], we define the set $\Omega$ as

$$
\Omega=\left\{(q, p): \dot{W}(q, p)=G^{\top} \nabla_{p} H_{d}=G^{\top} M_{d}^{-1}(q) p=0\right\} .
$$

Using

$$
M_{d}^{-1}=\frac{\Delta}{\Delta_{d}}\left[\begin{array}{cc}
m_{3} & \frac{\sigma \cos \left(q_{1}\right)\left(\cos \left(q_{1}\right)+\epsilon\right)}{\gamma} \\
\frac{\sigma \cos \left(q_{1}\right)\left(\cos \left(q_{1}\right)+\epsilon\right)}{\gamma} & \epsilon+\cos \left(q_{1}\right)
\end{array}\right],
$$

we obtain

$$
\begin{aligned}
G^{\top} M_{d}^{-1}(q) p & =\left[\begin{array}{ll}
0 & 1
\end{array}\right]\left(\frac{\Delta}{\Delta_{d}}\left[\begin{array}{cc}
m_{3} & \frac{\sigma \cos \left(q_{1}\right)\left(\cos \left(q_{1}\right)+\epsilon\right)}{\gamma} \\
\frac{\sigma \cos \left(q_{1}\right)\left(\cos \left(q_{1}\right)+\epsilon\right)}{\gamma} & \epsilon+\cos \left(q_{1}\right)
\end{array}\right]\right)\left[\begin{array}{l}
p_{1} \\
p_{2}
\end{array}\right]=0 \\
& =\frac{\Delta\left(\cos \left(q_{1}\right)+\epsilon\right)}{\gamma \Delta_{d}}\left(\sigma \cos \left(q_{1}\right) p_{1}+\gamma p_{2}\right)=0,
\end{aligned}
$$

which implies

$$
\sigma \cos \left(q_{1}\right) p_{1}+\gamma p_{2}=0
$$

as the term $\frac{\Delta\left(\cos \left(q_{1}\right)+\epsilon\right)}{\gamma \Delta_{d}}>0$ due to $\epsilon>1$. From (55), there are two cases to satisfy $\dot{H}_{d}=0$ : 
Case 1: $\cos \left(q_{1}\right)=\frac{k \pi}{2}$ with $k$ odd and $p_{2}=0$, or

Case 2: $p_{1}=p_{2}=0$.

We will now show that Case 1 is not feasible. Note that $p_{2}=0 \Longrightarrow \dot{p}_{2}=0$, and from the system dynamics (1) with (36) and (37), $\dot{p}_{2}$ is the control input (45), (47). That is

$$
\begin{aligned}
\dot{p}_{2}= & -\frac{\sigma \cos \left(q_{1}\right)}{\gamma}\left(\gamma m_{3}-\left(\rho+\gamma \sin ^{2}\left(q_{1}\right)\right)\left(\epsilon+\cos \left(q_{1}\right)\right)\right)\left(\frac{\mathcal{B}_{1}}{2} p_{1}^{2}+\mathcal{B}_{2} p_{1} p_{2}+\frac{\mathcal{B}_{3}}{2} p_{2}^{2}\right. \\
& \left.+\frac{\epsilon \lambda_{1} \lambda_{2}^{2} \sin \left(q_{1}\right)}{1-\lambda_{2}^{2} \cos ^{2}\left(q_{1}\right)}-\frac{\lambda_{1} \sin \left(q_{1}\right)}{\epsilon+\cos \left(q_{1}\right)}\right)-\left(\gamma m_{3}-\frac{\sigma^{2} \cos ^{2}\left(q_{1}\right)\left(\cos \left(q_{1}\right)+\epsilon\right)}{\gamma}\right) K_{p} q_{2} \\
& -j_{2} \frac{\Delta}{\gamma \Delta_{d}}\left(\gamma m_{3} p_{1}+\sigma \cos \left(q_{1}\right)\left(\cos \left(q_{1}\right)+\epsilon\right) p_{2}\right) \\
& -\frac{K_{v} \Delta\left(\cos \left(q_{1}\right)+\epsilon\right)}{\gamma \Delta_{d}}\left(\sigma \cos \left(q_{1}\right) p_{1}+\gamma p_{2}\right)=0 .
\end{aligned}
$$

Substituting $\cos \left(q_{1}\right)=\frac{k \pi}{2}($ with $k$ odd $)$ and $p_{2}=0$ yields

$$
j_{2} \frac{\Delta}{\gamma \Delta_{d}} \gamma m_{3} p_{1}=0
$$

which implies that $p_{1}=0 \Longrightarrow \dot{p}_{1}=0$. Now, from the system dynamics,

$$
\begin{aligned}
\dot{p}_{1}= & \frac{1}{2 \Delta^{2}}\left[\left(\rho+\gamma \sin ^{2}\left(q_{1}\right)\right) p_{1}^{2}+\gamma p_{2}^{2}+2 \sigma \cos \left(q_{1}\right) p_{1} p_{2}\right]\left(\left(\gamma^{2}+\sigma^{2}\right) \sin \left(2 q_{1}\right)\right) \\
& +\frac{1}{2 \Delta}\left[2 \sigma \sin \left(q_{1}\right) p_{1} p_{2}-\gamma \sin \left(2 q_{1}\right) p_{1}^{2}\right]+\kappa \sin \left(q_{1}\right)=0
\end{aligned}
$$

Note that because of the last term $\left(\kappa \sin \left(q_{1}\right)\right)$ in $(57), \dot{p}_{1}=0$ is only satisfied if $q_{1} \neq \frac{k \pi}{2}$ with $k$ odd. It is indeed satisfied if $q_{1}=0$. Thus, Case 1 cannot happen, and only Case 2 is true. Hence, the system can maintain the $\dot{H}_{d}=0$ condition only at the equilibrium point $\left(q_{e}, 0\right)$, which proves that this equilibrium is 'almost' globally asymptotically stable.

Figure 7 depicts the energy evolution for the rotary pendulum for two initial pendulum angles, $45^{\circ}$ and $180^{\circ}$. It shows that the system converges to its stable manifold corresponding to its isolated minimum energy. It also illustrates the fact that for the closed-loop system, the total energy function $H_{d}$ and the potential energy function $V_{d}$ satisfy the relation $V_{d}(t)<H_{d}(t)<H_{d}(0), \forall t$.

\subsection{Simulation results}

We present some simulation results obtained for the model of Quanser QUBEServo rotary pendulum shown in Figure 6 with the IDA-PBC controller (45), (47). The closed-loop performance is evaluated with two sets of simulations. In 

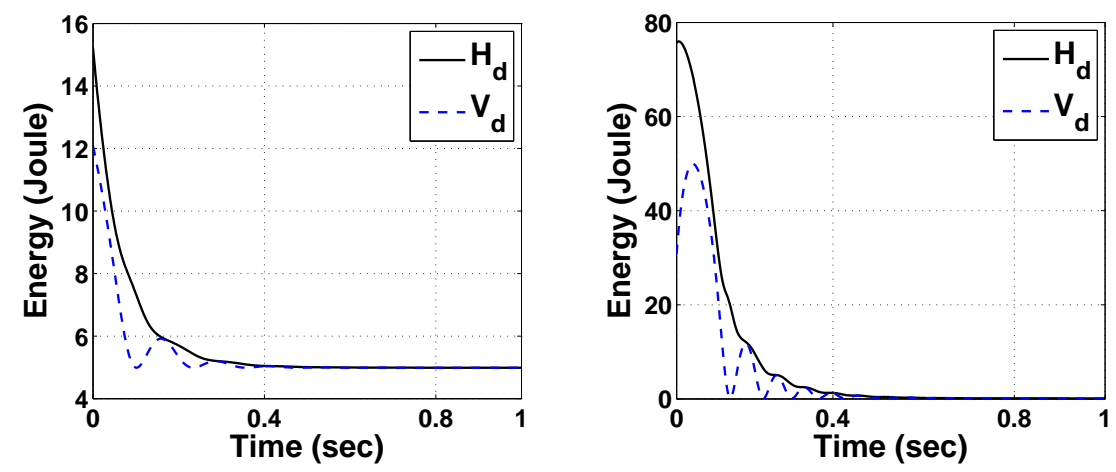

Fig. 7. The total energy of the rotary inverted pendulum. Left: $q_{1_{0}}=45^{\circ}$. Right: $q_{1_{0}}=180^{\circ}$.

the first set, the pendulum starts at $\left(q_{1_{0}}=\frac{\pi}{4}\right)$ and the controller parameters $K_{p}=0.01, K_{v}=1.7 \times 10^{-5}, K_{j}=2 \times 10^{-5}, m_{3}=50, \epsilon=1.1$. This $q_{1_{0}}$ is more than twice the initial angular position of the pendulum that is recommended for the balancing experiment of this rotary pendulum hardware using a linear state feedback controller obtained via a pole placement design [27]. The results are depicted in Figures 8 and 9. As can be observed, the pendulum can easily be stabilized at its upright position using the proposed controller with very little effort as shown by the low value of the control effort.
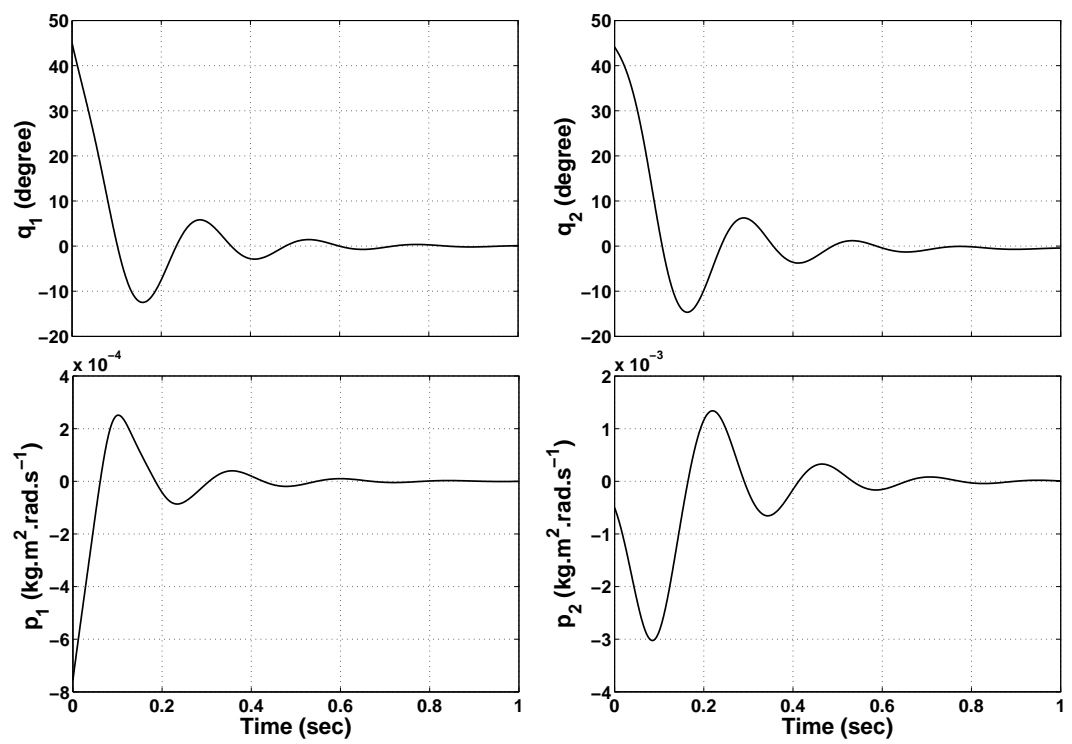

Fig. 8. Transient responses with $\left[q_{0}, p_{0}\right]=\left[\frac{\pi}{4}, 0.8,-0.7 \times 10^{-3},-0.5 \times 10^{-3}\right]$.

To show the global behaviour of the closed-loop system, simulations have been carried out with the pendulum swings up from the hanging position $\left(q_{1_{0}}=\pi\right)$, and having the parameters $K_{p}=6, K_{v}=2.1 \times 10^{-4}, K_{j}=1 \times 10^{-5}, m_{3}=54$, $\epsilon=1.3$. It is apparent from the Figure 10 that the proposed controller yields global asymptotic stabilization of the rotary inverted pendulum pendulum. However, It can be observed from Figure 11 that the control effort is significantly increased with the increased initial angular position of the pendulum. 


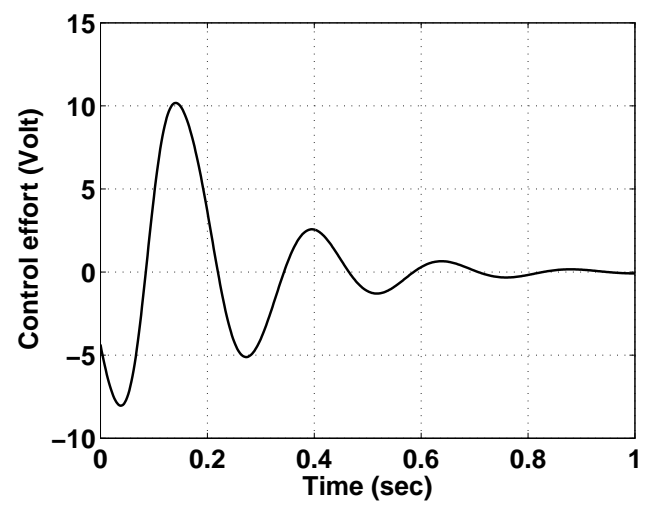

Fig. 9. Control input with $\left[q_{0}, p_{0}\right]=\left[\frac{\pi}{4}, 0.8,-0.7 \times 10^{-3},-0.5 \times 10^{-3}\right]$.

More simulations on a different rotary inverted pendulum pendulum hardware can be found in [28].
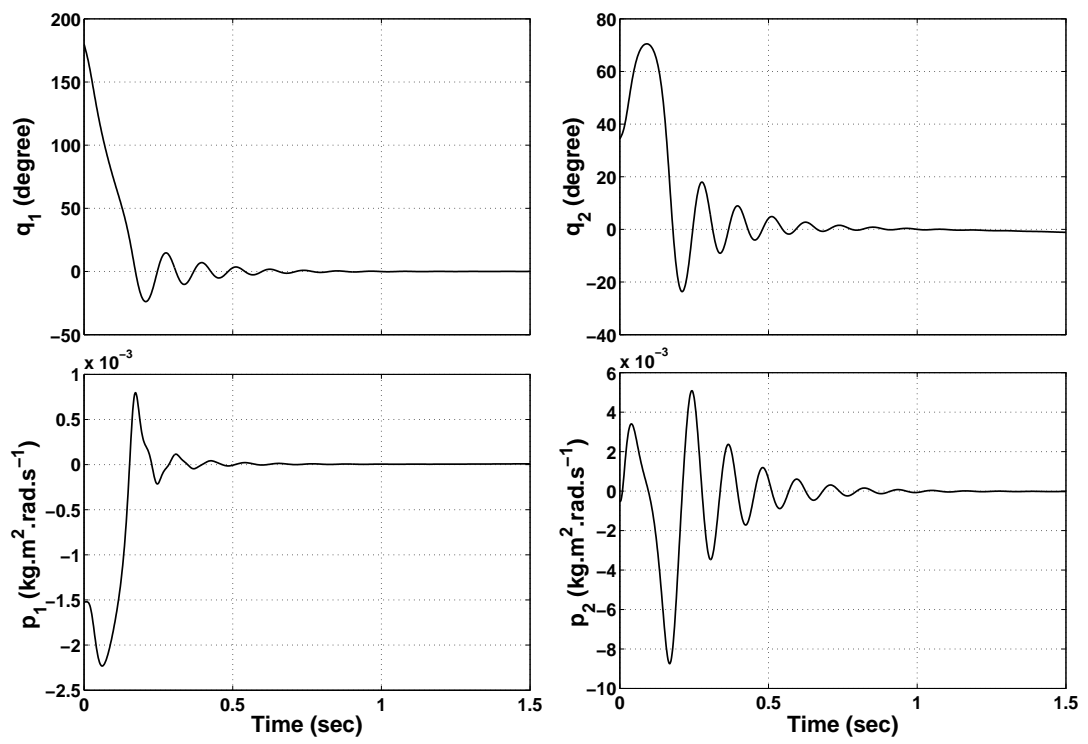

Fig. 10. Transient responses with $\left[q_{0}, p_{0}\right]=\left[\pi, 0.6,-1.5 \times 10^{-3},-0.5 \times 10^{-3}\right]$.

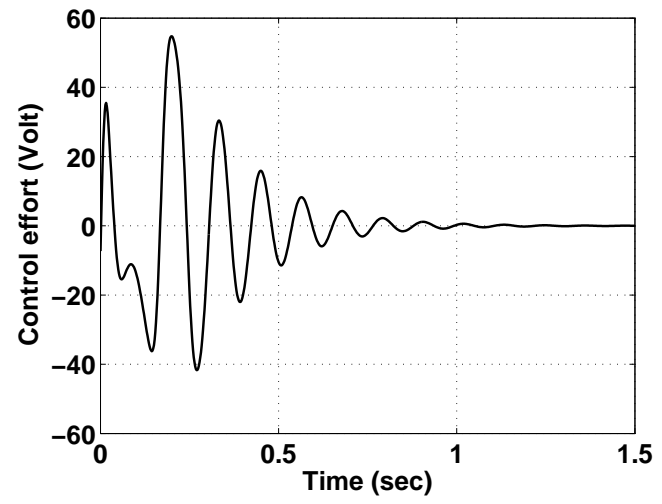

Fig. 11. Control input with $\left[q_{0}, p_{0}\right]=\left[\pi, 0.6,-1.5 \times 10^{-3},-0.5 \times 10^{-3}\right]$. 


\section{Hardware Experiments with the Rotary Inverted Pendulum}

\subsection{Experimental setup}

The experiments are also performed with the Quanser QUBE-Servo rotary pendulum. The hardware comprises an $18 \mathrm{~V}$ brushed DC motor contained in a solid aluminium frame and attached to the arm using magnets. Two singleended optical shaft encoders are used to measure the angular positions of the pendulum, $q_{1}$, and arm, $q_{2}$. The hardware is connected to a PC through the QUBE-Servo USB interface. This interface has its own built-in PWM voltagecontrolled power amplifier and data acquisition device. The output voltage range to the load is between $\pm 10 \mathrm{~V}$. The interaction between the $\mathrm{PC}$ and the hardware is driven by the QUARC real-time control software integrated with Matlab/Simulink to actuate the DC motor and read the angular positions $q_{1}$ and $q_{2}$. The sampling time of the control is 0.002 second.

\subsection{Friction compensation}

Note that the PCH modelling framework neglects some components of the dynamics to comply with the underlying concept of energy conservation in this modelling approach. For the rotary pendulum, the PCH model used in Section 5.1 does not consider non-conservative forces (e.g. friction) [1]. Thus, applying the controller (45)-(47) alone is not enough to stabilize the pendulum in the hardware implementation, due to the effects of friction which are not taken into account in the controller design. Friction is present in any mechanical systems and is a crucial aspect of many control systems. It can be highly nonlinear and may lead to steady-state errors, tracking errors, limit cycles and other undesirable behaviour [23]. Friction compensation is a common approach to deal with such effects and to achieve improved performance.

In this experiment, we have taken into account the Coulomb + viscous friction model, which has been widely used for friction compensation in many control systems applications (see $[33,30]$ ). The friction compensation term

$$
u_{f}=b\left(\dot{q}_{2}-\dot{q}_{1}\right)+k_{f} \operatorname{sign}\left(\dot{q}_{2}-\dot{q}_{1}\right)
$$

with $k_{f}$ the friction coefficient and $b$ the viscous damping coefficient of the link, is added to the IDA-PBC controller (45), (47) in order to overcome/compensate friction and hence enhance the performance of the closed-loop system. Considering the friction in the joints and taking into account the unmodelled dynamics introduced by the encoder cable as shown in Figure 6, the best friction model's parameters are estimated as $k_{f}=0.5755$ and $b=0.005755$. Thus, the total control law applied to the hardware is of the form

$$
u=u_{e s}+u_{d i}+u_{f} .
$$




\subsection{States measurement}

The two optical shaft encoders that measure the angular positions of the pendulum and the arm have the resolution of 2048 counts per revolution in quadrature mode (512 lines per revolution). The momenta $p_{1}$ and $p_{2}$ are obtained from the relation $p=M \dot{q}$, where the angular velocities $\dot{q}_{1}$ and $\dot{q}_{2}$ of the pendulum and arm are obtained by differentiating their corresponding measured angular positions. A low-pass filter has been added to the output of each differentiator to remove some high-frequency components (noise) which appears as a result of quantization due to the encoder measurement. The lowpass filters have been set as $50 /(s+50)$, with the cutoff frequency $w_{f}=50$ $\mathrm{rad} / \mathrm{sec}$ or $w_{f}=50 /(2 \pi)=7.96 \mathrm{~Hz}$.

\subsection{Experimental results}

In this section, we show our experimental results on the QUBE- rotary pendulum system. The total control law (59) has been applied to stabilize the pendulum at its upward position. The initial condition for the position of the pendulum was $q_{1_{0}}=45^{\circ}$. We will discuss later in this paper the factors that limited achieving a larger (DoA). However, this DoA still far larger than what was obtained using the linear controllers (the maximum achieved using a linear controller given in [27] was $\left.q_{1_{0}}=20^{\circ}\right)$.

In the experiment, the pendulum was set to start from a downward position. A swing-up controller based on the strategy developed in [5] has been applied to drive the pendulum up to the initial angle $\left(q_{1_{0}}\right)$. Once the pendulum reaches this angle, the controller switches to the IDA-PBC stabilizing controller. The controller parameters used in the experiment were $m_{3}=67, K_{p}=8.0 \times 10^{-4}$, $K_{v}=6 \times 10^{-6}$ and $\epsilon=1$. Note that while in simulation we can set the initial values of every state, this is not the case for the experiment. The only state for which the initial value can be set directly is the pendulum angle $q_{1_{0}}$.

Figures 12 and 13 show the experimental results for a hybrid controller (i.e. swing-up and stabilizing) for the inverted pendulum. In order to clearly show the effect of our proposed controller, we have magnified the parts of these figures starting from the instant of commutation $(t=6.16 \mathrm{sec})$ i.e. the instant in which the switching to IDA-PBC stabilizing controller take place as shown in Figures 14 and 15.

We can observe from Figure 14, which depicts the time histories of the positions and velocities of the pendulum and the arm, that all states converge to their desired equilibrium point, thus achieving asymptotic stabilization of the closed-loop system with the controller (59). Furthermore, this figure shows the smooth and fast convergence of the states while the pendulum and arm 
exhibit slight oscillatory behaviour ( $<1.5$ degrees). The profile of the control input is shown in Figure 15. We can observe the smooth control effort but with a more demanding effort to balance the pendulum at its vertical upward position.
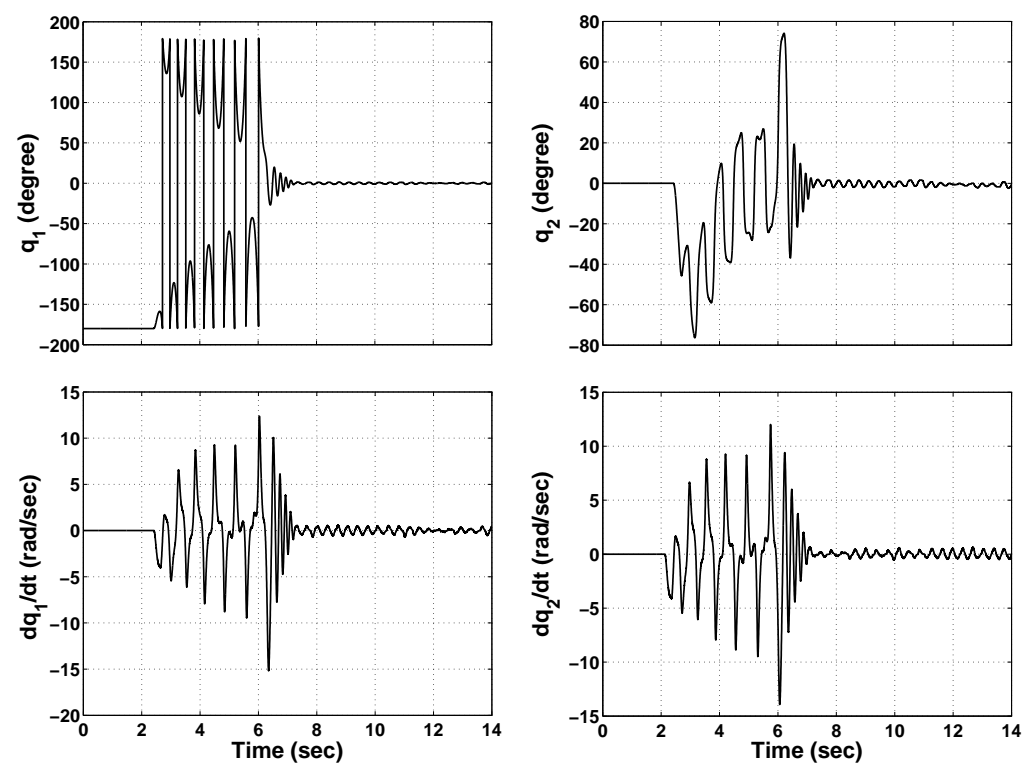

Fig. 12. Experimental results (swing-up and stabilization): state trajectories of the rotary pendulum system.

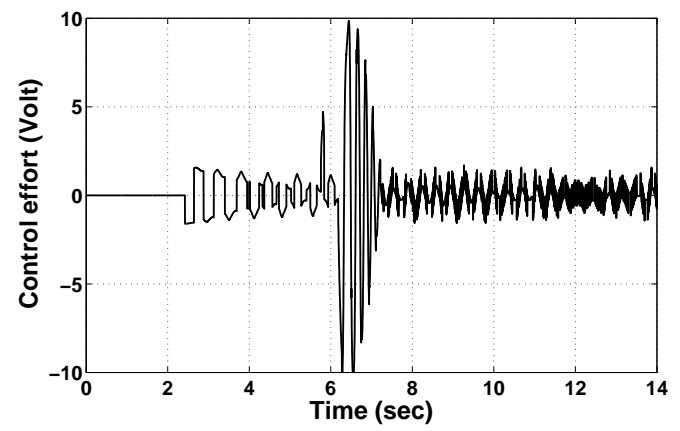

Fig. 13. Experimental results (swing-up and stabilization): control input.

\subsection{Robustness of the proposed IDA-PBC}

In order to show the robustness of the proposed controller, the pendulum has been perturbed, by slightly pushing it, at $t=14.5 \mathrm{sec}$. The experimental results are shown in Figures 16 and 17. As we can observe, the states recover from the injected disturbance, converging fast and smoothly to their desired values. While the trajectories of the states $q_{1}, \dot{q}_{1}$ and $\dot{q}_{2}$ converge to their exact desired values, $q_{2}$ which is the arm position converges to another position. This is expected as each position of the arm is an equilibrium. Furthermore, Figure 17 shows that the control effort in response for the perturbation is smooth 

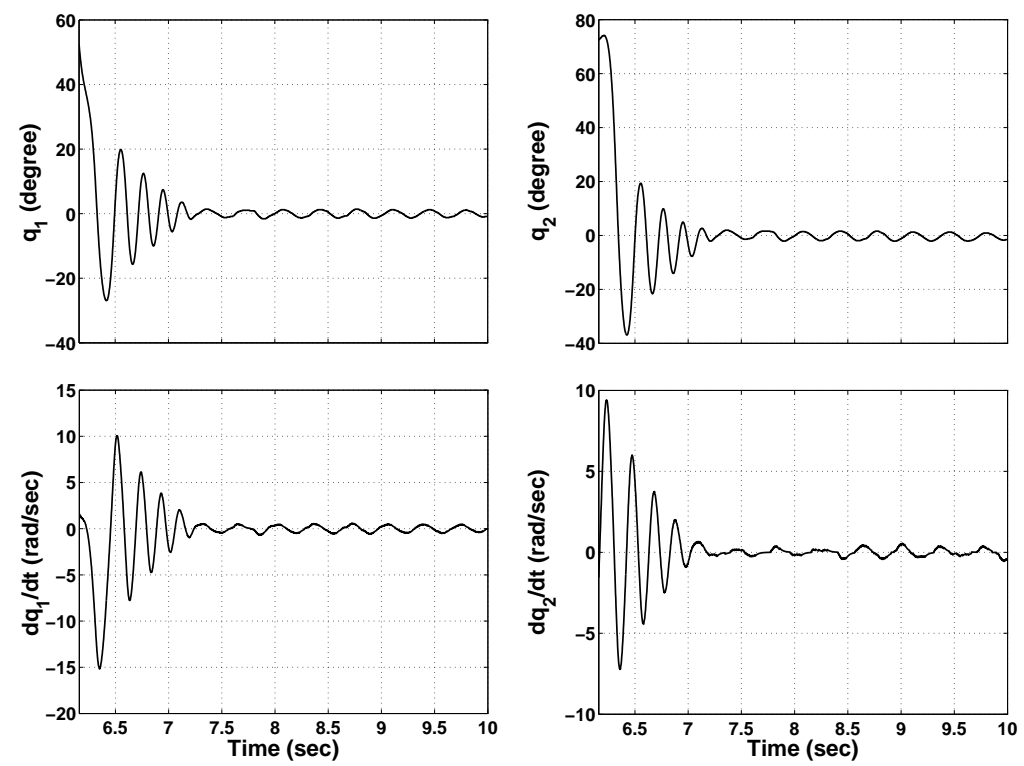

Fig. 14. Experimental results (stabilization): state trajectories of the rotary pendulum system.

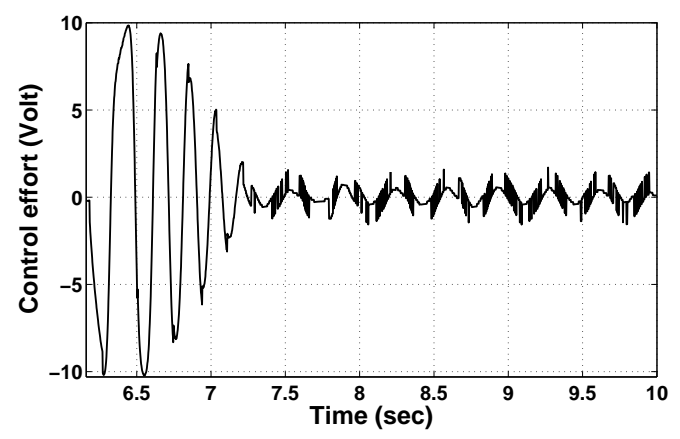

Fig. 15. Experimental results (stabilization): control input.

and remains within the acceptable voltage range. It is evident from these graphs that, the closed-loop system is robust with respect to disturbances on the pendulum. Although in theory, global asymptotic stabilization can be achieved, the maximum value of the pendulum angle achieved in experimental results was $q_{1_{0}}=45^{\circ}$. This can be mainly attributed to hardware limitations; 1) saturation of the control input; The motor is relatively small and provides insufficient torque to enlarge the initial pendulum angle, thus the domain of attraction. As shown in Figure 15, $v_{\max }$ was required already to stabilize the system starting from $q_{1_{0}}=45^{\circ} .2$ ) The arm does not rotate a full $360^{\circ}$ by design: the encoder cable is attached from the pendulum module encoder to the Encoder 1 connector on the top panel of the QUBE-Servo, hence a stopper is used to avoid any contact between the pendulum and the cable as shown in Figure 6. 

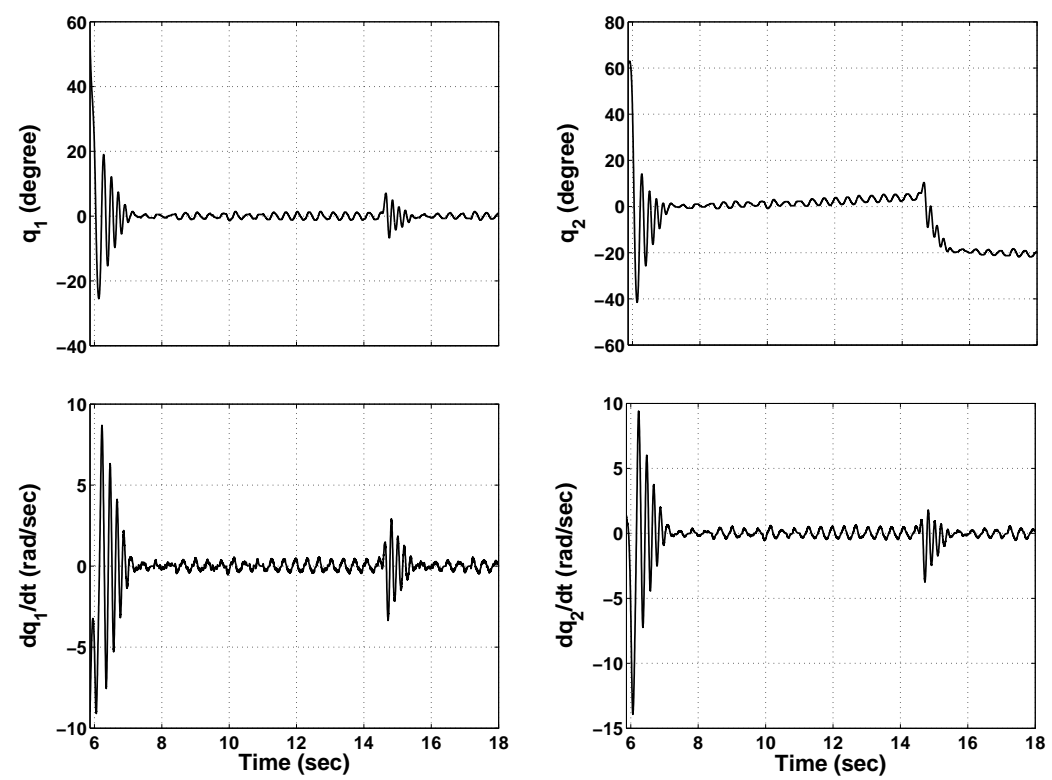

Fig. 16. Experimental results (robustness): state trajectories of the rotary pendulum system.

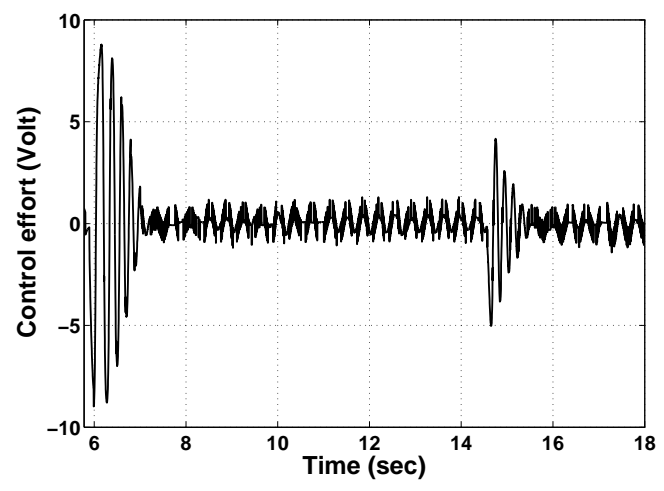

Fig. 17. Experimental results (robustness): control input.

\section{Conclusion and Future Works}

We have improved the IDA-PBC method for under-actuated mechanical systems. This improvement is achieved via a particular simplification of the matching PDEs. Solving these PDEs is the main difficulty in application of this method. We have defined a general construction procedure to reparametrize the inertia matrix and used to simplify and solve potential energy PDEs, achieving total energy shaping which is essential for stabilization of UMSs. The result has been successfully applied to solve an almost global stabilization control design for an inertia wheel pendulum and a rotary inverted pendulum which belong to two groups of PCH systems, the separable and non-separable systems, respectively. The proposed method has significantly simplified the design computation and also yield a simpler form of the controller for both systems. It has been also shown by realistic simulations that this design re- 
sults in a very high closed-loop performance of these systems in full nonlinear dynamics.

Furthermore, we have presented a successful experimental implementation of this approach to the rotary inverted pendulum hardware. The results have proved the effectiveness of the controller and its robustness with respect to disturbances. The theoretical results presented in this paper (together with [28]) and the experimental results can be used as the motivation to use this method in other real engineering applications. Current research is under way to extend this approach for a larger class of underactuated mechanical systems, as well as proposing an observer that facilitate the IDA-PBC methodology.

\section{References}

[1] J.A. Acosta. Furuta's pendulum: A conservative nonlinear model for theory and practise. Mathematical Problems in Engineering, 2010:29, 2010.

[2] J.A. Acosta, R. Ortega, A. Astolfi, and A.D. Mahindrakar. Interconnection and damping assignment passivity based control of mechanical systems with underactuation degree one. IEEE Trans. Automatic Control, 50:1936-1955, 2005.

[3] D. Angeli. Almost global stabilization of the inverted pendulum via continuous state feedback. Automatica, 37:1103-1108, 2001.

[4] K.J. Aström, J. Aracil, and F. Gordillo. A family of smooth controllers for swinging up a pendulum. Automatica, 44:1841-1848, 2008.

[5] K.J. Aström and K. Furuta. Swing up a pendulum by energy control. Automatica, 36:287-295, 2000.

[6] D. Auckly and L. Kapitanski. On the $\lambda$-equations for matching control laws. SIAM Journal on Control and Optimization, 41:1372-1388, 2002.

[7] G. Blankenstein, R. Ortega, and A.J. van der Schaft. The matching conditions of controlled Lagrangians and IDA-passivity based control. International Journal of Control, 75:645-665, 2002.

[8] A.M. Bloch, N.E. Leonard, and J.E. Marsden. Stabilization of the pendulum on a rotor arm by the method of controlled Lagrangians. In Proc. IEEE International Conference on Robotics and Automation, volume 1, pages 500-505 vol.1, 1999.

[9] A.M. Bloch, N.E. Leonard, and J.E. Marsden. Controlled Lagrangians and the stabilization of mechanical systems. I. The first matching theorem. IEEE Trans. Automatic Control, 45(12):2253-2270, 2000.

[10] D.J. Block, K.J. Aström, and M.W. Spong. The Reaction Wheel Pendulum. Morgan \& Claypool Publishers, 2007. 
[11] D.E. Chang and R.G. McLenaghan. Geometric criteria for the quasilinearization of the equations of motion of mechanical systems. IEEE Trans. Automatic Control, 58(4):1046-1050, 2013.

[12] I. Fantoni and R. Lozano. Nonlinear Control for Underactuated Mechanical Systems. Springer, 2002.

[13] I. Fantoni and R. Lozano. Stabilization of the Furuta pendulum around its homoclinic orbit. International Journal of Control, 75(6):390-398, 2002.

[14] F. Gomez-Estern, R. Ortega, F. Rubio, and J. Aracil. Stabilization of a class of underactuated mechanical systems via total energy shaping. IEEE Conf. on Decision and Control, 41:1372-1388, 2001.

[15] H.K. Khalil. Nonlinear Systems, 3nd Ed. Prentice Hall, 2002.

[16] P. Kotyczka and I. Sarras. On the equivalence of two nonlinear control approaches: Immersion and Invariance and IDA-PBC. European Journal of Control, 19(6):445 - 453, 2013.

[17] D.S. Laila and A. Astolfi. Discrete-time IDA-PBC design for separable Hamiltonian system. In Proc. IFAC World Congress, pages 838-843, 2005.

[18] A.D. Lewis. Potential energy shaping after kinetic energy shaping. In Decision and Control, 2006 45th IEEE Conference on, pages 3339-3344, Dec 2006.

[19] Y. Liu and H. Yu. A survey of underactuated mechanical systems. Control Theory Applications, IET, 7(7):921-935, May 2013.

[20] A.D. Mahindrakar, A. Astolfi, R. Ortega, and G. Viola. Further constructive results on interconnection and damping assignment control of mechanical systems: the acrobot example. Int. J. Robust Nonlinear Control, 16:671-685, 2006 .

[21] S. Nair and N.E. Leonard. A normal form for energy shaping: application to the Furuta pendulum. In Proc. 41st IEEE Conf. on Decision and Control, pages $516-521,2002$.

[22] R. Olfati-Saber. Nonlinear control of underactuated mechanical systems with application to robotics and aerospace vehicles. PhD thesis, MIT, Dept. of EECS, 2001.

[23] H. Olsson, K.J. Aström, C. Canudas de Wit, M. Gäfvert, and P. Lischinsky. Friction models and friction compensation. European Journal of Control, 4(3):176 - 195, 1998.

[24] R. Ortega and E. Garcia-Canseco. Interconnection and damping assignment passivity-based control: A survey. European Journal of Control, 10:432-450, 2004 .

[25] R. Ortega, M. Spong, F. Gomez-Estern, and G. Blankenstein. Stabilization of a class of underactuated mechanical systems via interconnection and damping assignment. IEEE Trans. Automatic Control, 47:1218 - 1233, 2002. 
[26] R. Ortega, A.J. van der Schaft, B. Maschke, and G. Escobar. Interconnection \& damping assignment passivity-based control of port-controlled Hamiltonian systems. Automatica, 38:585-596, 2002.

[27] Quanser Inc. QUBE-servo rotary pendulum user manual and workbook, 2014.

[28] M. Ryalat and D.S. Laila. IDA-PBC for a class of underactuated mechanical systems with application to a rotary inverted pendulum. In Proc. 52nd IEEE Conf. on Decision and Control, pages 5240-5245, 2013.

[29] J. Sandoval, R. Ortega, and R. Kelly. Interconnection and damping assignment passivity-based control of the pendubot. In Proc. IFAC World Congress, pages 7700-7704, 2008.

[30] I. Sarras, A.J. Acosta, R. Ortega, and A.D. Mahindrakar. Constructive immersion and invariance stabilization for a class of underactuated mechanical systems. Automatica, 49(5):1442 - 1448, 2013.

[31] M. Spong. Partial feedback linearization of underactuated mechanical systems. In Proc. IEEE/RSJ/GI Int. Conf. on Intelligent Robots and Systems, volume 1, pages 314-321, 1994.

[32] M. Spong. Energy based control of a class of underactuated mechanical systems. In Proc. IFAC World Congress, pages 431-435, 1996.

[33] M. Spong, D.J. Block, and K.J. Astrom. The mechatronics control kit for education and research. In Proc. IEEE International Conference on Control Applications, pages 105-110, 2001.

[34] M. Spong, P. Corke, and R. Lozano. Nonlinear control of the inertia wheel pendulum. Automatica, 37:1845-1851, 1999.

[35] J. van der Burg, R. Ortega, J. Scherpen, J.A. Acosta, and H.B. Siguerdidjane. An experimental application of total energy shaping control: Stabilization of the inverted pendulum on a cart in the presence of friction. In Proc. European Control Conference, 2007.

[36] A.J. van der Schaft. Stabilization of Hamiltonian systems. Nonlinear Analysis: Theory, Methods \&5 Applications, 10(10):1021 - 1035, 1986.

[37] A. Venkatraman, R. Ortega, I. Sarras, and A.J. van der Schaft. Speed observation and position feedback stabilization of partially linearizable mechanical systems. IEEE Trans. Automatic Control, 55(5):1059-1074, 2010.

[38] G. Viola, R. Ortega, R. Banavar, J.A. Acosta, and A. Astolfi. Total energy shaping control of mechanical systems: Simplifying the matching equations via coordinate changes. IEEE Trans. Automatic Control, 52:1093-1099, 2007. 